\title{
Synthesis, Optical Characteristics and Complex Formation of Molecular Receptors Based on 1,8-Naphthalimide Derivatives in Solution and in Composition of Hybrid Tin Dioxide Nanoparticles
}

\author{
Antonina N. Arkhipova, ${ }^{a}$ Pavel A. Panchenko, ${ }^{\mathrm{a}, \mathrm{b}}$ Nikolai E. Schepel, ${ }^{\mathrm{a}}$ \\ Yuri V. Fedorov, ${ }^{a}$ Marina N. Rumyantseva, ${ }^{\mathrm{c}}$ Artem V. Marikutsa, ${ }^{\mathrm{c}}$ \\ Alexander M. Gaskov, ${ }^{c}$ and Olga A. Fedorova ${ }^{a, b, c} @$ \\ a A.N. Nesmeyanov Institute of Organoelement Compounds of Russian Academy of Sciences, 119991 Moscow, Russia \\ ${ }^{\mathrm{b}}$ D.I. Mendeleev University of Chemisal Technology of Russia, 125047 Moscow, Russia \\ ${ }^{\mathrm{c}}$ M.V. Lomonosov Moscow State University, Chemistry Department, 119992 Moscow, Russia \\ @Corresponding authorE-mail: fedorova@ineos.ac.ru
}

\begin{abstract}
In the present work the synthesis of $N$-alkyl- and $N$-phenyl-1,8-naphthalimides containing dithiacrown ether, carboxyl group or both of these substituents has been developed. Molecular receptor 4-methoxy-N-benzodithiacrown-1,8-naphthalimide was synthesized from anhydride of 4-nitro-1,8-naphthalene dicarboxylic acid by acylation of crown-containing aniline and the replacement of nitro group onto methoxy one. Carboxylic derivatives of 1,8-naphthalimide with or without thiacrown ether moiety were prepared and the method of their covalent attachment to the $\mathrm{SnO}_{2}$ surface has been proposed. Molecular receptor 4-methoxy-N-benzodithiacrown-1,8-naphthalimide demonstrates selectivity towards $\mathrm{Hg}^{2+}$ and $\mathrm{Pb}^{2+} \mathrm{cations}^{2}$ in acetonintrile solution and towards $\mathrm{Hg}^{2+}$ in aqueous media. Due to its structure this receptor shows low fluorescence intensity in the free state and substantial increase of fluorescence in the presence of $\mathrm{Hg}^{2+}$ and $\mathrm{Pb}^{2+}$ is observed. This phenomenon is explained by the fact that an efficient photoinduced electron transfer takes place between fluorophore and receptor parts of the molecule in the absence of metal ions, resulting in fluorescence quenching. Electron transfer process is blocked in the presence of metal ions because the lone pair of electrons originally involved in the electron transfer process is engaged in metal ion binding. As a result the fluorescence enhancement is observed upon complexation with metal ions. 1,8-Naphthalimide derivatives in the composition of hybrid $\mathrm{SnO}_{2}$ sol demonstrate optical characteristics similar to those of molecular fluorophores. However, the binding of metal ions by hybrid nanoreceptor was not observed. It should be due to effective interaction of crown-containing molecule with $\mathrm{SnO}_{2}$ surface.
\end{abstract}

Keywords: 1,8-Naphthalimide, dithiacrown ether, tin dioxide, complex formation, fluorescence, nanoparticle.

\section{Синтез, оптические свойства и комплексообразование молекулярных рецепторов на основе производных 1,8-нафталимида в растворе и в составе гибридных наночастиц АиоксиАа олова}

\author{
А. Н. Архипова, ${ }^{a}$ П. А. Панченко, ${ }^{a}{ }^{a b}$ Н. Э. Шепель, ${ }_{1}^{a}$ Ю. В. Федоров, ${ }^{a}$ \\ М. Н. Румянцева, ${ }^{c}$ А. В. Марикуца, ${ }^{c}$ А. М. Гаськов, ${ }^{c}$ О. А. Федорова ${ }^{\mathrm{a}, \mathrm{b}, \mathrm{c} @}$ \\ а Институт Элементоорганических Соединений им. А.Н. Несмеянова РАН, 119991 Москва, Россия

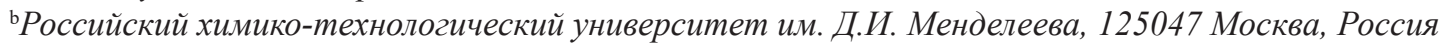

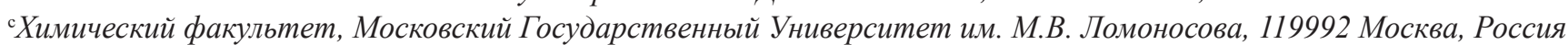 \\ ${ }^{\circledR}$ E-mail: fedorova@ineos.ac.ru
}


В настоящем исследовании синтезировань производные 1,8-нафталимида, содержащие в качестве заместителей дитиакраун-эфир фрагмент и карбоксильные группь. Наличие карбоксильных групп позволило осуществить пришивку нафталимида на поверхность наночастиц диоксида олова. Изучено комплексообразование краун-содержащих молекулярных 1,8-нафталимидов в растворе и в составе наночастиц.

Ключевые слова: 1,8-Нафталимид, тиакраун-эфир, диоксид олова, комплексообразование, флуоресценция, наночастицы.

\section{Introduction}

Determination of metal cations and hydrogen ions in the environment and biological systems is a major technical challenge for industry, medicine, ecology, as well as for chemical and biochemical research. Among the large arsenal of modern physical and chemical methods of analysis the optical spectroscopy has huge popularity, ${ }^{[1]}$ which is primarily due to the relative simplicity of the experiment coupled with a high sensitivity with respect to detectable substrates. In the past two decades, much progress has been achieved due to the development of optical chemosensors containing a selective receptor, and a signaling moiety capable of changing its spectral characteristics as a result of complex formation. ${ }^{[2]}$

The functionalization of nanostructured solids with specific groups to enhance active functions, such as the recognition of guests or to switch surface properties, is particularly interesting. Such materials with a high and readily accessible specific surface can amplify certain functional chemical processes. The step from a onedimensional molecule to a two-dimensional arrangement - the hetero-supramolecular ensemble - leads to unique properties which are not simply an extrapolation of the solution conduct to the surface. Hybrid semiconductor organic-inorganic materials are promising for the creation of sensor materials. They combine the inorganic semiconductor basis providing an electrical detection of the analyte and the organic modifier responsible for chemical reaction with the analyte. ${ }^{[3]}$ A critical step is the rational choice and preparation of the sensing material, because most of the important properties of a sensor, such as, for example, sensitivity and selectivity, strongly depend on the characteristics of the sensing material. For this reason a large effort has been done from one side to prepare materials having promising characteristics for sensor applications and from the other side to understand their sensing mechanisms, with the aim to improve their sensing performances, in terms of stability, selectivity, sensitivity.

Following this idea, the aim of the work presented here is to study the possibility to develop hybrid materials composed by $\mathrm{SnO}_{2}$ and dithiacrown ether containing 1,8-naph- thalimides (Scheme 1). Nanoscale particles of tin dioxide are of great scientific and practical interest. Such characteristics as transparency, semiconducting properties and relatively low cost, provide the application of the tin dioxide as components of optoelectronic devices and materials. Thus, the tin dioxide is widely used as an electrode material in solar cells, component of light-emitting diodes, liquid crystal displays, transistors and so-called "smart windows" as well as an active material in gas sensor equipment. ${ }^{[4]}$

One of the most popular platforms for the creating of optical molecular sensor device is the imide of naphthalic acid (1,8-naphthalimide). Derivatives of 1,8-naphthalimide are an important class of organic luminophores, which are widely used as optical brighteners, ${ }^{[5]}$ laser dyes,${ }^{[6]}$ solar cells elements, ${ }^{[7]}$ fluorescent markers in biology, ${ }^{[8]}$ electroluminescent materials, ${ }^{[9]}$ anticancer drugs, ${ }^{[10]}$ fluoroscent crack detection reagents. ${ }^{[11]}$ The 1,8-naphthalimide derivatives demonstrate a large variety of photophysical properties and can act as the photoactive components of the optical chemosensors. Furthermore, the 1,8-naphthalimide derivatives containing dithiacrown ether moieties are capable of binding with heavy and transition metals cations in organic solvents and in aqueous medium. ${ }^{[12]}$

Previously we have carried out the attachment of crown ether containing 1,8-naphthalimides to the surface of $\mathrm{SnO}_{2}$ through the silicon-organic linker. ${ }^{[13]}$ The obtained hybrid material showed a fluorescence enhancement upon the binding with magnesium cations. In the present paper the attachment of 1,8-naphthalimide derivatives to the surface of $\mathrm{SnO}_{2}$ nanoparticles have been done by applying of carboxylic group or chloro anhydride groups incorporated in the $N$-imide fragment of studied compounds (Scheme 1) and superficial $\mathrm{OH}$ groups of tin dioxide. In literature there are only a few examples of the organic-inorganic material composed by $\mathrm{SnO}_{2} \cdot{ }^{[13-15]}$

\section{Experimental}

NMR spectra were recorded on a Bruker Avance 300, 400 and $600 \mathrm{MHz}$ instruments operating at 300.13, 400.13, 600.22 MHz (for ${ }^{1} \mathrm{H}$ ) and $75.47,100.61,150.93 \mathrm{MHz}$ (for ${ }^{13} \mathrm{C}$ ) respectively.
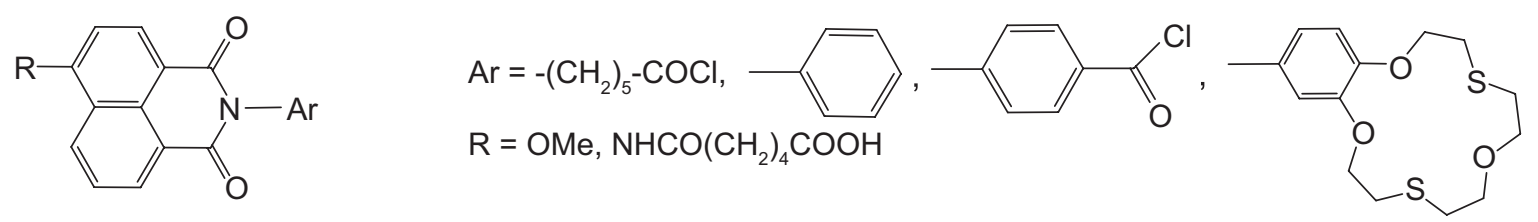

Scheme 1. 
The measurements were performed in $\mathrm{CD}_{3} \mathrm{COCD}_{3}, \mathrm{CD}_{3} \mathrm{SOCD}_{3}$ and $\mathrm{CD}_{3} \mathrm{CN} ; \delta$ values are given in ppm, coupling constants are presented in Hz. The chemical shifts were determined with an accuracy of $0.01 \mathrm{ppm}$ relative to the signals corresponding to the residual solvent and recalculated to the internal standard (TMS); the spin-spin coupling constants $(J)$ were measured with an accuracy of $0.1 \mathrm{~Hz}$. The assignment of ${ }^{13} \mathrm{C}$ signals is based on the data of twodimensional NMR spectra (HMBC and HSQC). The numbering of carbon atoms in the naphthalimide moiety, $N$-aryl ring and crown ether fragment of compounds commonly used by us for the description of ${ }^{1} \mathrm{H}$ and ${ }^{13} \mathrm{C}$ NMR spectra is shown on Figures 3, 4.

Melting points were determined in capillaries using Meltemp instrument and were not corrected. Elemental analysis was performed in the microanalysis laboratory of A.N. Nesmeyanov Institute of Organoelement compounds of RAS. The reaction course and purity of the final products was followed by TLC on silica gel DC-Alufolien Kieselgel 60 F254 plates (Merck). Column chromatography was carried out using silica gel Kieselgel 60 (particle size 0.063-0.200 mm, Merck) and benzene-ethanol mixture as an eluent.

Mass spectra were obtained on an Agilent 1100 Series LC/ MSD trap interface operated in positive-ion mode. The analyte solution was injected directly into the device. The flow rate was $400 \mu \mathrm{l} / \mathrm{h}$, the drying gas temperature was $350{ }^{\circ} \mathrm{C}$ and the pressure was $10 \mathrm{psi}$. The nebulizer needle voltage was $4.5-5.5 \mathrm{kV}$. The isotope distribution was calculated using the Molecular Weight Calculator software, version 6.73 .

Electronic absorption spectra were measured on a twochannel spectrophotometer Varian-Cary 100, fluorescence spectra were recorded on spectrofluorimeter FluoroLog-3-221 at $20 \pm 1{ }^{\circ} \mathrm{C}$. The observed fluorescence was detected at a direct angle relative to the excitation beam in the case of determining the quantum yields of compounds and frontally relative to the exciting beam in the case of measuring sol fluorescence. The fluorescence spectra were corrected for the nonuniformity of detector spectral sensitivity. Spectroscopic grade solvents (Aldrich, Acros) were used for recording of the absorption and fluorescence spectra.

A study by FTIR absorption spectroscopy was performed on a spectrometer Perkin Elmer Spectrum One. The spectra were recorded in transmission mode in wavenumber range $4000-370 \mathrm{~cm}^{-1}$ in steps of $1 \mathrm{~cm}^{-1}$. Samples (dried powders sols) weighing $5 \mathrm{mg}$ were grinded with $100 \mathrm{mg}$ of potassium bromide (Aldrich, «for FTIR analysis») and pressed into tablets of about $0.5 \mathrm{~mm}$ thick and $12 \mathrm{~mm}$ diameter.

General Procedure for acylation of aromatic amines by 4-substituted 1,8-naphthalic anhydride. To a suspension of $10.0 \mathrm{~mol}$ of 4-bromo- or 4-nitronaphthalic anhydride in $30 \mathrm{ml}$ of glacial acetic acid or ethyl alcohol $10.0-20.0 \mathrm{mmol}$ of the appropriate amine was added. The reaction mixture was refluxed for 6 hours, cooled to room temperature. The precipitate of $N$-substituted naphthalimide was filtered off, washed with $5 \%$ hydrochloric acid, then with $10 \%$ aqueous $\mathrm{Na}_{2} \mathrm{CO}_{3}$, water and ethanol. The product was dried at $80{ }^{\circ} \mathrm{C}$.

6-Nitro-2-phenyl-1H-benzo[d,e]isoquinoline-1,3(2H)-dione (4-nitro-N-phenyl-naphthalimide) (1a). According to the general procedure for acylation of aromatic amines $1.2 \mathrm{~g}$ (91\% yield) of 4-nitro- $N$-phenylnaphthalimide $2 \mathrm{a}$ was obtained from $1.0 \mathrm{~g}$ (4.1 $\mathrm{mmol})$ of 4-nitronaphthalic anhydride, $0.75 \mathrm{ml}(8.2 \mathrm{mmol})$ of aniline and $12 \mathrm{ml}$ of acetic acid. M.p. $291-293{ }^{\circ} \mathrm{C}$ (lit. ${ }^{[16]} 280$ $\left.282{ }^{\circ} \mathrm{C}\right) .{ }^{1} \mathrm{H}$ NMR $\left(300.13 \mathrm{MHz},\left[\mathrm{D}_{6}\right] \mathrm{DMSO}, 21^{\circ} \mathrm{C}\right) \delta \mathrm{ppm}(\mathrm{J} / \mathrm{Hz})$ : 7.38-7.45 (m, 2H, H(10), H(14)), 7.45-7.59 (m, 3H, H(11), H(12), $\mathrm{H}(13)), 8.13$ (dd, 1H, H(6), J=7.2, J=8.6), 8.59 (d, 1H, H(3), J=8.0), 8.62 (d, $1 \mathrm{H}, \mathrm{H}(2), J=8.0), 8.65$ (d, $1 \mathrm{H}, \mathrm{H}(7), J=7.2), 8.77$ (d, $1 \mathrm{H}$, $\mathrm{H}(5), J=8.6)$. MS, $m / z(\mathrm{I}, \%): 318$ ([M]+) (76), 317 (100), 287 (25), 271 (42), 227 (21), 214 (8), 195 (9), 179 (11), 151 (9), 125 (9).

6-Nitro-2-(2,3,5, 6,8,9,11,12-octahydrobenzo[b][1,4,10,7,13] trioxadithiacyclopentadecyn-15-yl)-1H-benzo[d,e]isoquinoline1,3(2H)-dione $(\mathbf{l b})$. According to the general procedure for acylation of aromatic amines $460 \mathrm{mg}$ (yield $71 \%$ ) of 4-nitro- $N$ arylnaphthalimide $\mathbf{2 b}$ was obtained from $300 \mathrm{mg}(1.2 \mathrm{mmol})$ of 4-nitronaphthalic anhydride, $650 \mathrm{mg}(1.8 \mathrm{mmol})$ of the aniline hydrochloride salt containing dithiacrown-ether, $160 \mathrm{mg}(2.0 \mathrm{mmol})$ of sodium acetate and $10 \mathrm{ml}$ of acetic acid. M.p. $228-230{ }^{\circ} \mathrm{C}$ (lit. $\left.{ }^{[17]} 228-230{ }^{\circ} \mathrm{C}\right) .{ }^{1} \mathrm{H}$ NMR $\left(300.13 \mathrm{MHz},\left[\mathrm{D}_{6}\right] \mathrm{DMSO}, 23{ }^{\circ} \mathrm{C}\right)$ $\delta \mathrm{ppm}(\mathrm{J} / \mathrm{Hz}): 2.85-3.11\left(\mathrm{~m}, 8 \mathrm{H}, \mathrm{CH}_{2}(16), \mathrm{CH}_{2}(17), \mathrm{CH}_{2}(20)\right.$, $\left.\left.\mathrm{CH}_{2}(21)\right), 3.68-3.78\left(\mathrm{~m}, 4 \mathrm{H}, \mathrm{CH}_{2}(18), \mathrm{CH}_{2}(19)\right), \mathrm{CH}_{2}(15)\right)$, $4.22-4.30\left(\mathrm{~m}, 2 \mathrm{H}, \mathrm{CH}_{2}(22)\right), 6.95$ (dd, $\left.1 \mathrm{H}, \mathrm{H}(14), J=2.2, J=8.6\right)$, 7.08 (d, 1H, H(10), J=2.2), 7.11 (d, 1H, H(13), J=8.6), 8.13 (dd, 1H, $\mathrm{H}(6), J=7.4, J=8.6), 8.58$ (d, 1H, H(3), $J=8.0$ ), 8.62 (d, 1H, H(2), $J=8.0), 8.64$ (d, 1H, H(7), $J=7.4), 8.76(\mathrm{~d}, 1 \mathrm{H}, \mathrm{H}(5), J=8.6)$. Found (\%): C, 57.79; H, 4.46; N, 5.14; S, 11.62. $\mathrm{C}_{26} \mathrm{H}_{24} \mathrm{~N}_{2} \mathrm{O}_{7} \mathrm{~S}_{2}$. Calculated (\%): C, 57.76; H, 4.47; N, 5.18; S, 11.84

6-Methoxy-2-phenyl-1H-benzo[d,e]isoquinoline-1,3(2H)-dione (4-methoxy-N-phenyl-naphthalimide) (2a). 4-Nitro- $N$-phenylnaphthalimide $1 \mathrm{a}(50 \mathrm{mg}, 0.16 \mathrm{mmol})$ and $32 \mathrm{mg}(0.32 \mathrm{mmol})$ of potassium carbonate were dissolved in $4 \mathrm{ml}$ of methanol. The mixture was refluxed for $6 \mathrm{~h}$, then it was poured into $8 \mathrm{ml}$ of water and acidified with $4 \mathrm{ml}$ of acetic acid. The resulting precipitate was filtered off, washed with water and dried to yield $39 \mathrm{mg}(80 \%)$ of 4-methoxy- $N$ - phenylnaphthalimide 2a. M.p. $234-238{ }^{\circ} \mathrm{C}$ (lit. [18]: $\left.235-239^{\circ} \mathrm{C}\right) .{ }^{1} \mathrm{H}$ NMR $\left(300.13 \mathrm{MHz},\left[\mathrm{D}_{6}\right]\right.$ acetone, $\left.21^{\circ} \mathrm{C}\right) \delta$ ppm $(\mathrm{J} / \mathrm{Hz}): 4.22$ (s, 3H, $\left.\mathrm{OCH}_{3}\right), 7.32-7.42$ (m, 3H, H(11), H(12), $\mathrm{H}(13)), 7.45$ (d, 1H, H(3), J=8.0), 7.48-7.56 (m, 2H, H(10), H(14)), 7.85 (dd, $1 \mathrm{H}, \mathrm{H}(6), J=7.3, J=8.6), 8.50-8.58$ (m, 2H, H(5), H(7)), 8.64 (d, $1 \mathrm{H}, \mathrm{H}(2), J=8.0$ ). Found (\%): C, 75.19; H, 4.30; N, 4.59. $\mathrm{C}_{19} \mathrm{H}_{13} \mathrm{NO}_{3}$. Calculated (\%): C, 75.22; H, 4.32; N, 4.62.

6-Methoxy-2-(2, 3, 5, 6, 8, 9, 11,12-octahydrobenzo [b] [1,4,10,7,13]trioxadithiacyclopentadecyn-15-yl)-1H-benzo[d,e] isoquinoline-1,3(2H)-dione (2b). A solution containing $138 \mathrm{mg}$ $(2.55 \mathrm{mmol})$ of sodium methylate and $7 \mathrm{ml}$ of methanol was added to a suspension of $100 \mathrm{mg}(0.17 \mathrm{mmol})$ of 4-nitronaphthalimide $\mathbf{1 b}$ in $10 \mathrm{ml}$ of methanol. The reaction mixture was refluxed for $10 \mathrm{~h}$ and then cooled to room temperature. Methanol was removed in vacuum, $10 \mathrm{ml}$ of water was added to the residue. The precipitate of 4-methoxy- $N$-arylnaphthalimide $\mathbf{2 b}$ was filtered off, washed with water and ethanol to yield $73 \mathrm{mg}(90 \%)$ of $\mathbf{2 b}$. M.p. $233-235^{\circ} \mathrm{C}$. ${ }^{1} \mathrm{H}$ NMR $\left(600.22 \mathrm{MHz},\left[\mathrm{D}_{6}\right] \mathrm{DMSO}, 2{ }^{\circ} \mathrm{C}\right) \delta \mathrm{ppm}(\mathrm{J} / \mathrm{Hz}): 2.84$ $3.11\left(\mathrm{~m}, 8 \mathrm{H}, \mathrm{CH}_{2}(16), \mathrm{CH}_{2}(17), \mathrm{CH}_{2}(20), \mathrm{CH}_{2}(21)\right), 3.65-3.81(\mathrm{~m}$, $\left.4 \mathrm{H}, \mathrm{CH}_{2}(18), \mathrm{CH}_{2}(19)\right), 4.06-4.14\left(\mathrm{~m}, 2 \mathrm{H}, \mathrm{CH}_{2}(15)\right), 4.16$ (s, 3H, $\left.\mathrm{OCH}_{3}\right), 4.22-4.31\left(\mathrm{~m}, 2 \mathrm{H}, \mathrm{CH}_{2}(22)\right), 6.89(\mathrm{dd}, 1 \mathrm{H}, \mathrm{H}(14), J=1.8$, $J=8.6), 7.04$ (d, 1H, H(10), J=1.8), 7.08 (d, 1H, H(13), $J=8.6), 7.35$ (d, 1H, H(3), J=8.2), 7.81-7.90 (m, 1H, H(6)), 8.47 (d, 1H, H(2), $J=8.2), 8.52(\mathrm{~d}, 1 \mathrm{H}, \mathrm{H}(7), J=7.3), 8.58(\mathrm{~d}, 1 \mathrm{H}, \mathrm{H}(5), J=8.5) .{ }^{13} \mathrm{C}$ NMR (150.93 MHz, [D $]$ DMSO, $\left.20{ }^{\circ} \mathrm{C}\right) \delta$ ppm: $32.30\left(\mathrm{CH}_{2} \mathrm{~S}\right)$, $32.40\left(\mathrm{CH}_{2} \mathrm{~S}\right), 32.73\left(\mathrm{CH}_{2} \mathrm{~S}\right), 32.75\left(\mathrm{CH}_{2} \mathrm{~S}\right), 57.02\left(\mathrm{OCH}_{3}\right), 71.64$ $\left(\mathrm{CH}_{2} \mathrm{O}\right), 71.69\left(\mathrm{CH}_{2} \mathrm{O}\right), 71.95\left(\mathrm{CH}_{2} \mathrm{O}\right), 71.97\left(\mathrm{CH}_{2} \mathrm{O}\right), 106.88$ $(\mathrm{C}(3)), 113.86$ (C(13)), 115.05 (C(10)), 115.50 (C(1)), 122.01 (C(14)), $123.00(\mathrm{C}(8)), 123.14$ (C(4a)), 127.01 (C(6)), 129.21 (C(5)), $129.97(\mathrm{C}(8 \mathrm{a})), 130.02(\mathrm{C}(9)), 131.97(\mathrm{C}(7)), 133.98(\mathrm{C}(2))$, 147.52 (C(12)), 147.99 (C(11)), $160.06(\mathrm{C}(4)), 163.03(\mathrm{C}(8 \mathrm{~b}))$, 163.57 (C(8c)). MS (IES), calculated, $\mathrm{m} / z$ : 580.19 ; found: 580.13 $\left([\mathrm{M}+\mathrm{H}]^{+}\right)$. Found (\%): C, 62.21; H, 5.78; N, 7.16. $\mathrm{C}_{30} \mathrm{H}_{33} \mathrm{~N}_{3} \mathrm{O}_{5} \mathrm{~S}_{2}$. Calculated (\%): C, 62.15; H, 5.74; N, 7.25.

6-Amino-2-phenyl-1H-benzo[d,e]isoquinoline-1,3(2H)-dione (4-amino- $N$-phenylnaphthalimide) (3a). To a stirred suspension of $2.30 \mathrm{~g}(7.2 \mathrm{mmol})$ of 4-nitro- $N$-phenylnaphthalimide $1 \mathrm{a}$ in $34 \mathrm{ml}$ of ethanol at $50{ }^{\circ} \mathrm{C}$ a solution of $11.24 \mathrm{~g}$ of $\mathrm{SnCl}_{2} \cdot 2 \mathrm{H}_{2} \mathrm{O}$ in $8.0 \mathrm{ml}$ of concentrated hydrochloric acid $(\rho=1.18 \mathrm{~g} / \mathrm{ml})$ was added dropwise. The mixture was refluxed for 1.5 hours. The reaction mixture was poured into $50 \mathrm{ml}$ of water, the precipitate was filtered off, washed with water, $1 \%$ aqueous $\mathrm{NaOH}$ (to remove tin compounds), then again with water and ethanol. The product was dried at $80^{\circ} \mathrm{C}$ to give $1.90 \mathrm{~g}(92 \%$ yield $)$ of 3a. M.p. $310-312^{\circ} \mathrm{C}$ (lit. $\left.{ }^{[19]}: 302-304{ }^{\circ} \mathrm{C}\right) .{ }^{1} \mathrm{H}$ NMR $\left(300.13 \mathrm{MHz},\left[\mathrm{D}_{6}\right] \mathrm{DMSO}, 23^{\circ} \mathrm{C}\right) \delta \mathrm{ppm}(\mathrm{J} / \mathrm{Hz}): 6.87(\mathrm{~d}, 1 \mathrm{H}$, $\left.\mathrm{H}(3),{ }^{3} \mathrm{~J}=8.5\right), 7.26-7.35$ ( $\left.\mathrm{m}, 2 \mathrm{H}, \mathrm{H}(10), \mathrm{H}(14)\right), 7.37-7.57$ (m, 5H, $\left.\mathrm{H}(11), \mathrm{H}(12), \mathrm{H}(13), \mathrm{NH}_{2}\right), 7.63-7.72(\mathrm{~m}, 1 \mathrm{H}, \mathrm{H}(6)), 8.19$ (d, 1H, 
$\left.\mathrm{H}(2),{ }^{3} J=8.5\right), 8.42\left(\mathrm{~d}, 1 \mathrm{H}, \mathrm{H}(7),{ }^{3} J=7.2\right), 8.66\left(\mathrm{~d}, 1 \mathrm{H}, \mathrm{H}(5),{ }^{3} J=8.5\right)$. UV-Vis $\left(\mathrm{CH}_{3} \mathrm{CN}\right) \lambda_{\text {max }} \mathrm{nm}(\mathrm{lg} \varepsilon): 417$ (4.11). MS, $m / z$ (I, \%): 289 (16), $288\left([\mathrm{M}]^{+}\right)(81), 287$ (100), 244 (9), 243 (29), 242 (9), 216 (6), 195 (18), 167 (6), 140 (11).

6-Amino-2-(2,3,5,6,8,9,11,12-octahydrobenzo[b][1,4,10,7,13] trioxadithiacyclopentadecyn-15-yl)-1H-benzo[d,e]isoquinoline$1,3(2 \mathrm{H})$-dione $(\mathbf{3 b})$. To a solution of $150 \mathrm{mg}(0.278 \mathrm{mmol})$ of 4-nitronaphthalimide $\mathbf{1 b}$ in $4 \mathrm{ml}$ of DMF warmed to $90{ }^{\circ} \mathrm{C} 1.2 \mathrm{ml}$ of $100 \%$ hydrazine hydrate was added. ${ }^{[20]}$ Raney nickel catalyst prepared from $0.7 \mathrm{~g}$ of nickel aluminum alloy (50 weight $\%$ of $\mathrm{Ni}$ ) was added portionwise to the reaction mass at vigorous stirring over a period of $30 \mathrm{~min}$. The reaction mixture was kept at $90{ }^{\circ} \mathrm{C}$ for 1 hour, the presence of starting nitro compound was monitored by TLC $\left(\mathrm{SiO}_{2}\right.$ plates, eluting with $\left.\mathrm{PhH}-\mathrm{EtOH}, 5: 1\right)$. After the reaction mixture was cooled to room temperature, the catalyst was filtered off, the filtrate was evaporated in vacuo. The residue was purified by column chromatography (silica gel, eluent $\mathrm{PhH}-\mathrm{EtOH}$, gradient elution) to yield $23 \mathrm{mg}(16 \%)$ of $\mathbf{3 b}$. M.p. $249-250{ }^{\circ} \mathrm{C}$. ${ }^{1} \mathrm{H}$ NMR (300.13 MHz, [D $]$ DMSO, $\left.23{ }^{\circ} \mathrm{C}\right) \delta \mathrm{ppm}(\mathrm{J} / \mathrm{Hz}): 2.85-3.10(\mathrm{~m}, 8 \mathrm{H}$, $\left.\mathrm{CH}_{2}(16), \mathrm{CH}_{2}(17), \mathrm{CH}_{2}(20), \mathrm{CH}_{2}(21)\right), 3.67-3.79$ (m, 4H, $\mathrm{CH}_{2}(18)$, $\left.\mathrm{CH}_{2}(19)\right), 4.07-4.17$ (m, 2H, $\left.\mathrm{CH}_{2}(15)\right), 4.19-4.29$ (m, 2H, $\left.\mathrm{CH}_{2}(22)\right)$, 6.82 (dd, $\left.1 \mathrm{H}, \mathrm{H}(14),{ }^{4} J=2.2,{ }^{3} \mathrm{~J}=8.6\right), 6.86$ (d, $\left.1 \mathrm{H}, \mathrm{H}(3),{ }^{3} \mathrm{~J}=8.4\right), 6.95$ (d, $\left.1 \mathrm{H}, \mathrm{H}(10),{ }^{4} J=2.2\right), 7.05$ (d, $\left.1 \mathrm{H}, \mathrm{H}(13),{ }^{3} J=8.6\right), 7.49$ (br. s, $2 \mathrm{H}$, $\left.\mathrm{NH}_{2}\right), 7.63-7.72(\mathrm{~m}, 1 \mathrm{H}, \mathrm{H}(6)), 8.18\left(\mathrm{~d}, 1 \mathrm{H}, \mathrm{H}(2),{ }^{3} \mathrm{~J}=8.4\right), 8.42$ (d, $\left.1 \mathrm{H}, \mathrm{H}(7),{ }^{3} J=7.3\right), 8.65$ (d, $\left.1 \mathrm{H}, \mathrm{H}(5),{ }^{3} J=8.5\right) .{ }^{13} \mathrm{C}$ NMR $(100.62$ $\mathrm{MHz},\left[\mathrm{D}_{6}\right.$ ]DMSO, $\left.27{ }^{\circ} \mathrm{C}\right) \delta \mathrm{ppm}: 30.31\left(\mathrm{CH}_{2} \mathrm{~S}\right), 30.40\left(\mathrm{CH}_{2} \mathrm{~S}\right)$, $30.72\left(2 \times \mathrm{CH}_{2} \mathrm{~S}\right), 70.66\left(\mathrm{CH}_{2} \mathrm{O}\right), 70.69\left(\mathrm{CH}_{2} \mathrm{O}\right), 70.94\left(\mathrm{CH}_{2} \mathrm{O}\right), 70.98$ $\left(\mathrm{CH}_{2} \mathrm{O}\right), 107.92(\mathrm{C}(1)), 108.20(\mathrm{C}(3)), 112.95(\mathrm{C}(13)), 114.48(\mathrm{C}(10))$, 119.48 (C(4a)), $121.67(\mathrm{C}(14)), 122.33(\mathrm{C}(8)), 124.03(\mathrm{C}(6)), 129.43$ (C(9)), $129.66(\mathrm{C}(5)), 130.17(\mathrm{C}(8 \mathrm{a})), 131.10(\mathrm{C}(7)), 133.99$ (C(2)), $147.64(\mathrm{C}(12)), 148.16(\mathrm{C}(11)), 152.76(\mathrm{C}(4)), 163.29(\mathrm{C}(8 \mathrm{~b})), 164.15$ $(\mathrm{C}(8 \mathrm{c}))$. UV-Vis $\left(\mathrm{CH}_{3} \mathrm{CN}\right) \lambda_{\max } \mathrm{nm}(\lg \varepsilon): 417$ (4.13). MS (IES), calculated, $m / z(I, \%): 617.03\left([\mathrm{M}+\mathrm{Ag}]^{+}\right)(92), 619.02\left([\mathrm{M}+\mathrm{Ag}+2]^{+}\right)$ (100); found: $617.08\left([\mathrm{M}+\mathrm{Ag}]^{+}\right)(92), 618.97\left([\mathrm{M}+\mathrm{Ag}+2]^{+}\right)(100)$. Found (\%): C, 61.32; H, 5.18; N, 5.45. $\mathrm{C}_{26} \mathrm{H}_{26} \mathrm{~N}_{2} \mathrm{O}_{5} \mathrm{~S}_{2}$. Calculated (\%): C, 61.16; H, 5.13; N, 5.49 .

6-(1,3-Dioxo-2-phenyl-2,3-dihydro-1H-benzo[d,e]isoquinolin-6-yl-amino)-6-adipic acid (4a). To a stirring suspension of $80 \mathrm{mg}$ $(0.28 \mathrm{mmol})$ of 4-aminonaphthalimide $3 \mathbf{a}$ in $0.8 \mathrm{ml}$ of pyridine at room temperature a solution of $0.19 \mathrm{~g}$ of monochloroanhydride of adipic acid in $0.8 \mathrm{ml}$ of DMF was added dropwise. The reaction mixture was stirred for 4 hours at $40-60{ }^{\circ} \mathrm{C}$, then the precipitate was filtered off, washed with water, $5 \%$ hydrochloric acid, again with water and hot ethanol. The product was dried in air to yield $69 \mathrm{mg}(60 \%)$ of 4a. M.p. $210-213^{\circ} \mathrm{C} .{ }^{1} \mathrm{H}$ NMR (400.22 MHz, [D $]$ DMSO, $\left.21^{\circ} \mathrm{C}\right) \delta \mathrm{ppm}(\mathrm{J} / \mathrm{Hz}): 1.57-1.72\left(\mathrm{~m}, 4 \mathrm{H}, \mathrm{CH}_{2}(25,26)\right), 2.29$ (t, 2H, $\left.\mathrm{CH}_{2}(24), J=7.5\right), 2.61\left(\mathrm{t}, 2 \mathrm{H}, \mathrm{CH}_{2}(23), J=7.5\right), 7.35-7.41$ (m, 2H, H(10), H(14)), 7.42-7.56 (m, 3H, H(11), H(12), H(13)), 7.93 (dd, $1 \mathrm{H}, \mathrm{H}(6), J=8.5, J=7.2), 8.33$ (d, $1 \mathrm{H}, \mathrm{H}(3), J=8.3), 8.49$ (d, 1H, H(2), J=8.3), 8.54 (d, 1H, H(7), J=7.2), 8.74 (d, 1H, H(5), $J=8.5$ ), 10.40 (br. s, $1 \mathrm{H}, \mathrm{NHCO}), 12.10$ (br. s, $1 \mathrm{H}, \mathrm{COOH}$ ). Found (\%): C, 69.28; H, 4.79; N, 6.71. $\mathrm{C}_{24} \mathrm{H}_{20} \mathrm{~N}_{2} \mathrm{O}_{5}$. Calculated (\%): C, $69.22 ; \mathrm{H}, 4.84 ; \mathrm{N}, 6.73$.

6-(2-(2,3,5,6,8,9,11,12-Octahydrobenzo [b] [1,4,10,7,13] trioxadithiacyclopentadecyn-15-yl)-1,3-dioxo-2,3-dihydro- $1 \mathrm{H}$ benzo[de]isoquinolin-6-yl-amino)-6-adipic acid (4b). To a stirring suspension of $85 \mathrm{mg}(1.67 \mathrm{mmol})$ of 4-aminonaphthalimide $\mathbf{3 b}$ in $1.0 \mathrm{ml}$ of pyridine at room temperature a solution of $0.20 \mathrm{~g}$ of monochloro anhydride of adipic acid in $1.0 \mathrm{ml}$ of DMF was added dropwise. The reaction mixture was stirred for 4 hours at $40-60{ }^{\circ} \mathrm{C}$, then diluted with water, the precipitate was filtered off, washed with $5 \%$ hydrochloric acid, again with water and ethanol several times. The product was dried in air to yield $71 \mathrm{mg}(67 \%)$ of $\mathbf{4 b}$. M.p. $183-186{ }^{\circ} \mathrm{C} .{ }^{1} \mathrm{H}$ NMR $\left(400.22 \mathrm{MHz},\left[\mathrm{D}_{1}\right] \mathrm{CDCl}_{3}, 22{ }^{\circ} \mathrm{C}\right) \delta \mathrm{ppm}(\mathrm{J} /$ $\mathrm{Hz}): 1.58-1.75\left(\mathrm{~m}, 4 \mathrm{H}, \mathrm{CH}_{2}(25,26)\right), 2.29\left(\mathrm{t}, 2 \mathrm{H}, \mathrm{CH}_{2}(24), J=7.5\right)$, 2.60 (t, 2H, $\left.\mathrm{CH}_{2}(23), J=7.5\right), 2.85-3.09$ (m, 8H, $\mathrm{CH}_{2}(16,17,20,21)$ ), 3.68-3.79 (m, 4H, $\left.\mathrm{CH}_{2}(18,19)\right), 4.07-4.18\left(\mathrm{~m}, 2 \mathrm{H}, \mathrm{CH}_{2}(15)\right), 4.19$ $4.33\left(\mathrm{~m}, 2 \mathrm{H}, \mathrm{CH}_{2}(22)\right), 6.67$ (d, 1H, H(13), J=8.8), 7.01-7.11 (m,
$2 \mathrm{H}, \mathrm{H}(10), \mathrm{H}(14)), 7.92$ (dd, 1H, H(6), J=8.8, J=7.2), 8.32 (d, 1H, $\left.\mathrm{H}(3),{ }^{3} J=8.4\right), 8.47$ (d, 1H, H(2), J=8.4), 8.53 (d, 1H, H(7), J=7.2), 8.73 (d, 1H, H(5), J=8.8), 10.39 (br.s, $1 \mathrm{H}, \mathrm{NHCO}$ ), 12.04 (br.s, $1 \mathrm{H}, \mathrm{COOH}$ ). Found (\%): C, 60.15; H, 5.24; N, 4.35. $\mathrm{C}_{32} \mathrm{H}_{34} \mathrm{~N}_{2} \mathrm{O}_{8} \mathrm{~S}_{2}$. Calculated (\%): C, 60.17; H, 5.37; N, 4.39.

6-(6-Nitro-1,3-dioxo-1H-benzo[d,e]isoquinoline-2-(3H)-yl) hexanoic acid (5). To a suspension of $0.08 \mathrm{~g}(0.3 \mathrm{mmol})$ of 4-nitro1,8-naphthalic anhydride 1 in $2 \mathrm{ml}$ of ethanol $0.04 \mathrm{~g}(0.3 \mathrm{mmol})$ of 6 -aminocaproic acid was added. The reaction mixture was refluxed for $2 \mathrm{~h}$, cooled to room temperature. The precipitate was filtered off, washed with $5 \%$ hydrochloric acid, water and ethanol to yield $0.04 \mathrm{~g}(53 \%)$ of 5. M.p. $168-170{ }^{\circ} \mathrm{C} .{ }^{1} \mathrm{H}$ NMR $\left(300.13 \mathrm{MHz},\left[\mathrm{D}_{6}\right]\right.$ DMSO, $\left.23{ }^{\circ} \mathrm{C}\right) \delta \mathrm{ppm}(\mathrm{J} / \mathrm{Hz}): 1.30-1.40,1.50-1.60,1.67-1.70(\mathrm{~m}$, $\left.6 \mathrm{H}, \mathrm{CH}_{2}(10), \mathrm{CH}_{2}(11), \mathrm{CH}_{2}(12)\right), 2.22$ (t, 2H, H(9), $\left.{ }^{3} J=7.3\right), 4.03$ (t, $\left.2 \mathrm{H}, \mathrm{CH}_{2}(13),{ }^{3} \mathrm{~J}=7.3\right), 8.09$ (dd, $\left.1 \mathrm{H}, \mathrm{H}(6),{ }^{3} \mathrm{~J}=7.5,{ }^{3} \mathrm{~J}=8.6\right), 8.56$ (d, 1H, H(2), $\left.{ }^{3} \mathrm{~J}=8.0\right), 8.58-8.65$ (m, 2H, H(5), H(7)), 8.71 (d, 1H, $\left.\mathrm{H}(3),{ }^{3} J=8.7\right)$. Found (\%): C, 60.65; H, 4.47; N, 7.88. $\mathrm{C}_{18} \mathrm{H}_{16} \mathrm{~N}_{2} \mathrm{O}_{6}$. Calculated (\%): C, 60.67; H, 4.53; N, 7.86.

6-(6-Methoxy-1,3-dioxo-1H-benzo[d,e]isoquinoline-2-(3H)$y$ l)hexanoic acid (6). $0.25 \mathrm{~g}(1.1 \mathrm{mmol})$ of 4-nitro-1,8-naphthalimido$N$-hexanoic acid 5 and $0.55 \mathrm{mg}(10.2 \mathrm{mmol})$ of sodium methylate were dissolved in $10 \mathrm{ml}$ of methanol. The reaction mixture was refluxed for $4 \mathrm{~h}$, cooled to room temperature, and $1 \mathrm{ml}$ of acetic acid was added to the reaction mixture, then the resulting solution was evaporated in vacuum. To the crude product water and $125 \mathrm{ml}$ of concentrated hydrochloric acid were added. The precipitate was filtered off and washed with water to yield $210 \mathrm{mg}(60 \%)$ of 6 . M.p. 160-162 ${ }^{\circ} \mathrm{C} .{ }^{1} \mathrm{H}$ NMR $\left(300.13 \mathrm{MHz},\left[\mathrm{D}_{6}\right] \mathrm{DMSO}, 21{ }^{\circ} \mathrm{C}\right) \delta \mathrm{ppm}$ $(\mathrm{J} / \mathrm{Hz})$ : $1.28-1.39\left(\mathrm{~m}, 2 \mathrm{H}, \mathrm{CH}_{2}(11)\right), 1.48-1.68\left(\mathrm{~m}, 4 \mathrm{H}, \mathrm{CH}_{2}(10)\right.$, $\left.\mathrm{CH}_{2}(12)\right), 2.21\left(\mathrm{t}, 2 \mathrm{H}, \mathrm{CH}_{2}(13),{ }^{3} \mathrm{~J}=7.4\right), 4.02$ (t, $2 \mathrm{H}, \mathrm{CH}_{2}(9)$, $\left.{ }^{3} J=7.4\right), 4.13\left(\mathrm{~s}, 3 \mathrm{H}, \mathrm{OCH}_{3}\right), 7.34\left(\mathrm{~d}, 1 \mathrm{H}, \mathrm{H}(3),{ }^{3} J=8.3\right), 7.83$ (dd, $\left.1 \mathrm{H}, \mathrm{H}(6),{ }^{3} J=7.5,{ }^{3} J=7.7\right), 8.48$ (d, $\left.1 \mathrm{H}, \mathrm{H}(2),{ }^{3} J=8.3\right), 8.51(\mathrm{~d}, 1 \mathrm{H}$, $\left.\mathrm{H}(7),{ }^{3} \mathrm{~J}=7.2\right), 8.55$ (d, $\left.1 \mathrm{H}, \mathrm{H}(5),{ }^{3} \mathrm{~J}=7.2\right), 11.99$ (s, $\left.1 \mathrm{H}, \mathrm{COOH}\right) .{ }^{13} \mathrm{C}$ NMR (100.61 MHz, [D ${ }_{6}$ DMSO, $\left.27^{\circ} \mathrm{C}\right) \delta$ ppm: $24.23\left(\mathrm{CH}_{2}\right), 27.33$ $\left(\mathrm{CH}_{2}\right), 33.48\left(\mathrm{CH}_{2}\right), 41.25\left(\mathrm{CH}_{2}\right), 56.67\left(-\mathrm{OCH}_{3}\right), 106.36(\mathrm{C}(3))$, $114.31(\mathrm{C}(1)), 121.98(\mathrm{C}(8)), 122.82(\mathrm{C}(4 \mathrm{a})), 126.49(\mathrm{C}(6)), 128.33$ $(\mathrm{C}(5)), 128.62(\mathrm{C}(8 \mathrm{a})), 131.12(\mathrm{C}(7)), 133.38(\mathrm{C}(2)), 160.38(\mathrm{C}(4))$, $162.99(\mathrm{C}(8 \mathrm{~b})), 163.61(\mathrm{C}(8 \mathrm{c})), 174.45(-\mathrm{COOH})$. Found (\%): $\mathrm{C}$, $66.80 ; \mathrm{H}, 5.62 ; \mathrm{N}, 4.14 ; \mathrm{O}, 23.43 . \mathrm{C}_{19} \mathrm{H}_{19} \mathrm{NO}_{5}$. Calculated (\%): C, $66.85 ; \mathrm{H}, 7.61 ; \mathrm{N}, 4.10 ; \mathrm{O}, 23.43$.

6-(6-Methoxy-1,3-dioxo-1H-benzo[d,e]isoquinoline-2-(3H)yl)hexyl chloride (7). 4-Methoxynaphthalimide 6 ( $0.1 \mathrm{~g}, 0.3 \mathrm{mmol})$ and $200 \mu \mathrm{l}(2.3 \mathrm{mmol})$ of oxalyl chloride were refluxed in $5 \mathrm{ml}$ of dichloromethane for 3 hours. The solvent was evaporated in vacuo. The obtained product $(0.14 \mathrm{~g})$ was used in the next step of synthesis without further purification.

2-(4-(6-Bromo-1,3-dioxo-1H-benzo[d, e]isoquinoline-2-(3H)$y l)$-phenyl) acetic acid (8). According to the general procedure for acylation of aromatic amines $980 \mathrm{mg}(69 \%)$ of 4-bromo- $N$-arylnaphthalimide $\mathbf{8}$ was obtained from $970 \mathrm{mg}$ (3.5 mmol) of 4-bromonaphthalic anhydride, $795 \mathrm{mg}(5.3 \mathrm{mmol})$ of 4-amino-phenylacetic acid and $27 \mathrm{ml}$ of acetic acid. M.p. 261$264{ }^{\circ} \mathrm{C} .{ }^{1} \mathrm{H}$ NMR $\left(600.22 \mathrm{MHz},\left[\mathrm{D}_{6}\right] \mathrm{DMSO}, 23{ }^{\circ} \mathrm{C}\right) \delta \mathrm{ppm}(\mathrm{J} / \mathrm{Hz})$ : 3.69 (s, 2H, $\left.\mathrm{CH}_{2}(15)\right), 7.29-7.35$ (m, 2H, H(10), H(14)), 7.37-7.44 (m, 2H, H(11), H(13)), 8.01 (dd, 1H, H(6), J=7.9, J=8.7), 8.21 (d, $1 \mathrm{H}, \mathrm{H}(3), J=8.1), 8.32$ (d, $1 \mathrm{H}, \mathrm{H}(2), J=8.1), 8.57-8.58$ (m, 2H, $\mathrm{H}(7), \mathrm{H}(5)) .{ }^{13} \mathrm{C}$ NMR $\left(150.93 \mathrm{MHz},\left[\mathrm{D}_{6}\right] \mathrm{DMSO}, 23{ }^{\circ} \mathrm{C}\right) \delta \mathrm{ppm}:$ $41.25\left(\mathrm{CH}_{2}\right), 106.56(\mathrm{C}(3)), 114.91(\mathrm{C}(1)), 122.98(\mathrm{C}(8)), 123.02$ $(\mathrm{C}(4 \mathrm{a})), 126.49(\mathrm{C}(6)), 128.13(\mathrm{C}(5)), 128.89$ (C(10), C(14)), 129.62 (C(8a)), $130.56(\mathrm{C}(11), \mathrm{C}(13)), 132.91$ (C(7)), 133.78 (C(2)), 134.72 (C(9)), 135.81 (C(12)), 161.38 (C(4)), 162.99 (C(8b)), 163.61 (C(8c)), 174.45 (-COOH). MS (IES), calculated, $m / z$ : 433.1233; found: $433.1235\left([\mathrm{M}+\mathrm{Na}]^{+}\right)$. Found (\%): C, 56.61; $\mathrm{H}, 3.34 ; \mathrm{N}, 3.06 ; \mathrm{C}_{20} \mathrm{H}_{12} \mathrm{BrNO}_{4} \times 0.8 \mathrm{CH}_{3} \mathrm{COOH}$. Calculated (\%): C, $58.56 ; \mathrm{H}, 2.95 ; \mathrm{N}, 3.41$.

2-(4-(6-Methoxy-1,3-dioxo-1H-benzo[d,e]isoquinoline-2(3H)-yl)-phenyl) acetic acid (9). $240 \mathrm{mg}(0.6 \mathrm{mmol})$ of 4-bromo$\mathrm{N}$-phenylnaphthalimide 8 and $70 \mathrm{mg}(1.2 \mathrm{mmol})$ of potassium 
carbonate were dissolved in $7 \mathrm{ml}$ of methanol. The reaction mixture was refluxed for 6 hours, then poured into $15 \mathrm{ml}$ of water and acidified with $7 \mathrm{ml}$ of acetic acid. The resulting precipitate was filtered off, washed with water and dried to yield $190 \mathrm{mg}(63 \%)$ of 9. M.p. $257-260{ }^{\circ} \mathrm{C} .{ }^{1} \mathrm{H}$ NMR $\left(600.22 \mathrm{MHz},\left[\mathrm{D}_{3}\right] \mathrm{CD}_{3} \mathrm{CN}, 25^{\circ} \mathrm{C}\right)$ $\delta$ ppm $(\mathrm{J} / \mathrm{Hz}): 3.69\left(\mathrm{~s}, 2 \mathrm{H}, \mathrm{CH}_{2}\right), 4.13\left(\mathrm{~s}, 3 \mathrm{H}, \mathrm{CH}_{3}\right), 7.21-7.30$ (m, 3H, H(10), H(14), H(3)), 7.43 (d, 2H, H(11), H(13)), 7.80 (dd, 1H, H(6), $J=7.9, J=8.7$ ), 8.47-8.56 (m, 2H, H(7), H(2)), 8.61 (d, $1 \mathrm{H}, \mathrm{H}(5), J=7.9) .{ }^{1} \mathrm{H}-\mathrm{NMR}\left(600.22 \mathrm{MHz},\left[\mathrm{D}_{6}\right] \mathrm{DMSO}, 24^{\circ} \mathrm{C}\right.$, $\delta / \mathrm{ppm}, J / \mathrm{Hz}): 3.35\left(\mathrm{~s}, 2 \mathrm{H}, \mathrm{CH}_{2}\right), 4.14\left(\mathrm{~s}, 3 \mathrm{H}, \mathrm{CH}_{3}\right), 7.10-7.16$ (m, 2H, H(10), H(14)), 7.27-7.38 (m, 3H, H(11), H(13), H(3)), 7.84 (dd, 1H, H(6), $J=7.9, J=8.7), 8.44-8.55$ (m, 2H, H(7), H(2)), 8.58 (d, $1 \mathrm{H}, \mathrm{H}(5), J=7.9) .{ }^{13} \mathrm{C}$ NMR $\left(150.93 \mathrm{MHz},\left[\mathrm{D}_{6}\right] \mathrm{DMSO}, 24{ }^{\circ} \mathrm{C}\right)$ $\delta$ ppm: $41.25\left(\mathrm{CH}_{2}\right), 56.67\left(-\mathrm{OCH}_{3}\right), 106.56(\mathrm{C}(3)), 114.91(\mathrm{C}(1))$, $122.98(\mathrm{C}(8)), 123.02(\mathrm{C}(4 \mathrm{a})), 126.49(\mathrm{C}(6)), 128.13(\mathrm{C}(5)), 128.89$ $(\mathrm{C}(10), \mathrm{C}(14)), 129.62(\mathrm{C}(8 \mathrm{a})), 130.56(\mathrm{C}(11), \mathrm{C}(13)), 132.91$ $(\mathrm{C}(7)), 133.78(\mathrm{C}(2)), 134.72(\mathrm{C}(9)), 135.81(\mathrm{C}(12)), 161.38(\mathrm{C}(4))$, $162.99(\mathrm{C}(8 \mathrm{~b})), 163.61$ (C(8c)), 174.45 (-COOH). MS (IES), calculated, $m / z$ : 384.0847 ; found: $384.0842\left([\mathrm{M}+\mathrm{Na}]^{+}\right)$. Found $(\%)$ : C, 61.52; H, 5.07; N, 3.51. $\mathrm{C}_{21} \mathrm{H}_{15} \mathrm{NO}_{5} \times 2.7 \mathrm{H}_{2} \mathrm{O}$. Calculated (\%): C, $61.50 ; \mathrm{H}, 5.02 ; \mathrm{N}, 3.42$.

2-(4-(6-Methoxy-1,3-dioxo-1H-benzo[de]isoquinoline2-(3H)-yl)-phenyl) acetyl chloride (10). $0.1 \mathrm{~g}$ (0.3 mmol) of 4-methoxynaphthalimide 9 was refluxed in $5 \mathrm{ml}$ of thionyl chloride for 3 hours. The excess of thionyl chloride was removed in vacuo. The obtained product $(0.15 \mathrm{~g})$ was used in the subsequent step without further purification.

Synthesis of $\mathrm{SnO}_{2}$ nanoparticles sols, modified by naphthalimide derivatives. All investigated sols based on $\mathrm{SnO}_{2}$ nanoparticles and naphthalimide were obtained by the following procedure. To the $100 \mathrm{ml}$ of $\mathrm{SnO}_{2}$ nanoparticles water sol in a dark flask $200 \mathrm{ml}$ of naphthalimide solution in DMSO with concentration of $5 \cdot 10^{-3} \mathrm{~mol} / 1$ of naphthalimide dye was added. The reaction mixture was stirred and kept at $60{ }^{\circ} \mathrm{C}$ for 1 hour, and then cooled to room temperature. Partly the obtained solution of modified sol was applied for further study of optical properties, and the remainder one was evaporated to dryness to carry out the study by IR spectroscopy. Samples were stored in a dark to avoid the naphthalimide degradation on the surface of $\mathrm{SnO}_{2}$ nanoparticles.

\section{Results and Discussion}

\section{Synthesis of 1,8-naphthalimide derivatives $\mathbf{2 a , b}$}

Synthesis of 4-methoxynaphthalimide derivatives containing $N$-phenyl (2a) and $N$-benzoditiacrown ether (2b) fragments is presented in Scheme 2. Compound 2a possesses the optical characteristics similar to those of $\mathbf{2} \mathbf{b}$ but is not able to coordinate with metal ions. The starting compound in the synthesis of $\mathbf{2 a , b}$ is commercially available 4-nitronaphthalic anhydride which is used to acylate the aromatic amines. For introduction of the donor methoxy group to the $4^{\text {th }}$ position of naphthalimide residue of $\mathbf{2 a , b}$ known procedures were applied..$^{[21]}$

Two-step synthesis was carried out to obtain $\mathbf{4 a , b}$. The first step was reduction of nitro groups of $\mathbf{1 a}, \mathbf{b}$ giving $\mathbf{3 a} \mathbf{a}, \mathbf{b}$. The second one was the interaction of amino derivatives $\mathbf{3 a}, \mathbf{b}$ with monochloro anhydride of adipic acid resulting in crown-free (4a) and dithiacrown-containing (4b) derivatives.

Synthesis of 4-methoxynaphthalimide derivatives 6 and 9 containing a carboxyl group in $N$-phenyl or $N$-alkyl substituent was carried out based on the 4-nitro- and 4-bromnaphthalic anhydride according to Scheme 4 (see

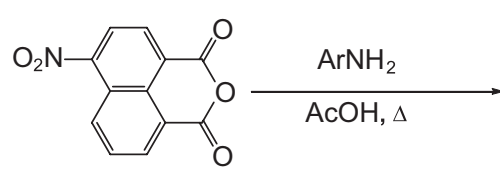

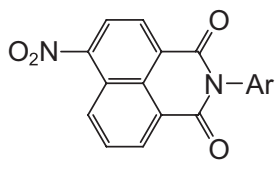

$1 \mathrm{a}, 91 \%$

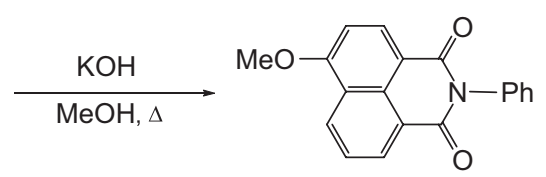

$2 a, 80 \%$
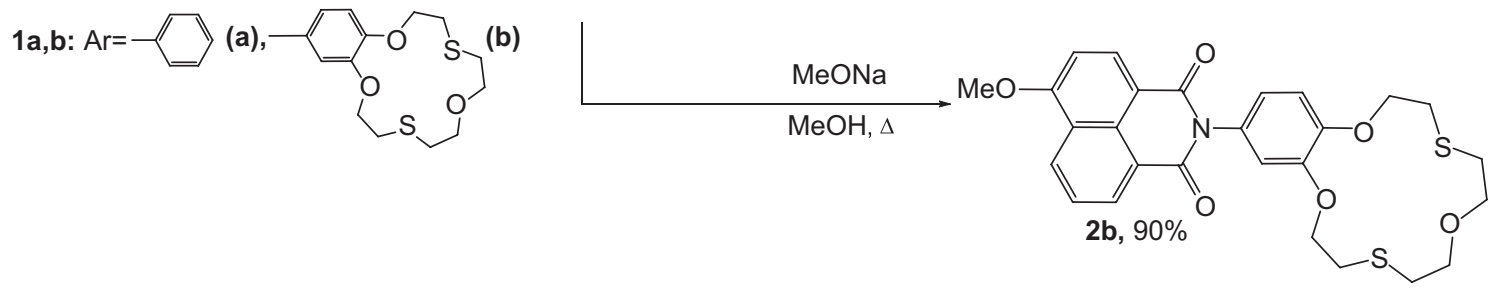

Scheme 2.

1a,b:

3a: $\mathrm{SnCl}_{2}, \mathrm{HCl}, \mathrm{EtOH}, \Delta$

3b: $\mathrm{N}_{2} \mathrm{H}_{4}$, Ni Raney, DMF, $\Delta$<smiles>Nc1ccc2c3c(cccc13)C(=O)N(Br)C2=O</smiles>

$3 a, 92 \%$

3 b, $16 \%$

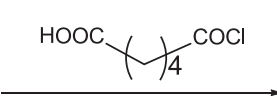

Py, DMF, $\Delta$

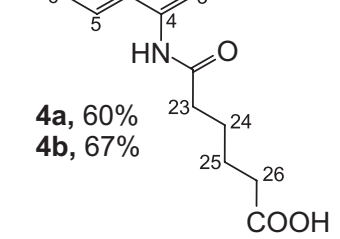

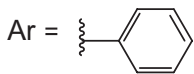

(a)

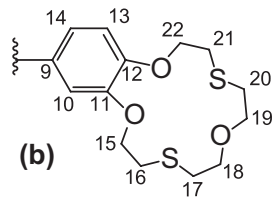

Scheme 3. 

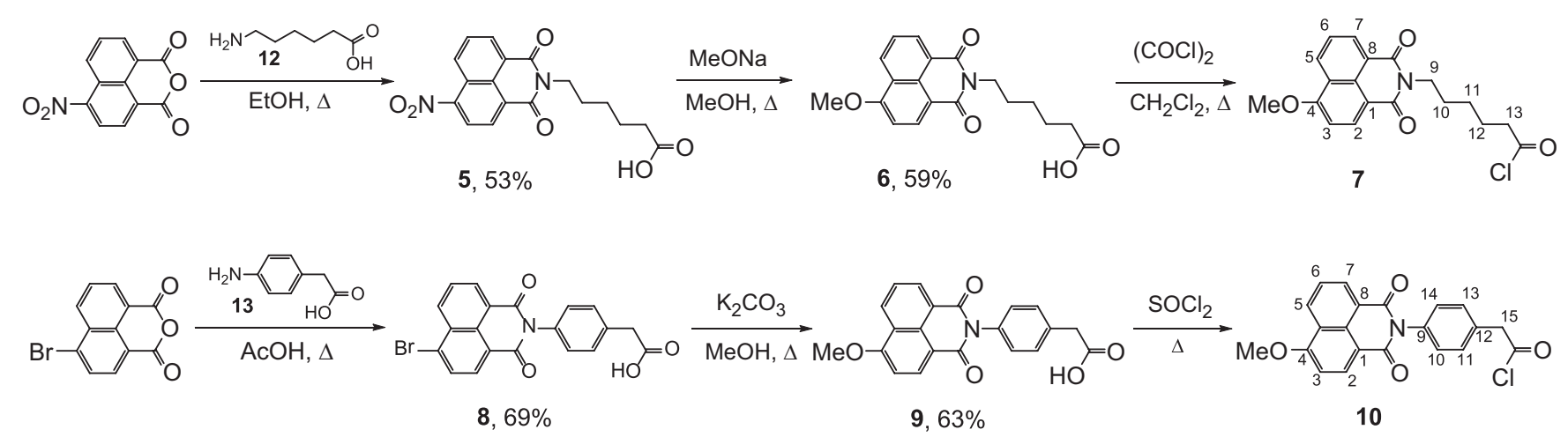

Scheme 4 .

the details in the Experimental Part). The variation of the carboxylic derivative structure was done aimed to find the better modification agent for $\mathrm{SnO}_{2}$.

\section{Preparation of the modified $\mathrm{SnO}_{2}$ nanoparticles}

An aqueous sol of $\mathrm{SnO}_{2}$ nanoparticles modified by naphthalimide derivatives were obtained and used for physico-chemical study. IR spectra of unmodified $\mathrm{SnO}_{2}$ nanoparticles and nanoparticles modified by organic components and starting naphthalimides $\mathbf{5 a}, \mathbf{b}, \mathbf{7}$ and $\mathbf{9}$ were recorded to confirm the covalent binding of compounds. Figures 1-3 show the IR spectra of $\mathrm{SnO}_{2}$, compound 9 and $\mathrm{SnO}_{2}$ nanoparticles modified by naphthalimide $\mathbf{9}$. The IR spectra of $\mathbf{5 a}, \mathbf{b}, 7$ and $\mathrm{SnO}_{2}$ nanoparticles modified by these compounds are presented in Supporting Information (SI, Figs. S2-S6). In the IR-spectrum of $\mathrm{SnO}_{2}$ nanoparticles the band of $\mathrm{O}-\mathrm{H}$ stretching in the region $3440-2500 \mathrm{~cm}^{-1}$ is observed (Figure 1). In IR spectrum of 9 there are bands belonging to carboxylate anion. There are intense band of antisymmetric stretching at $1650-1550 \mathrm{~cm}^{-1}$ and less intense symmetric one band at $1400 \mathrm{~cm}^{-1}$ (Figure 2). ${ }^{[22]}$ The broad band at $3400 \mathrm{~cm}^{-1}$ which is characteristic for the stretching of $\mathrm{O}-\mathrm{H}$ group is observed in the IR spectrum of molecular naphthalimide 9 (Figure 2). In the $\mathrm{SnO}_{2} \times \mathbf{9}$ hybrid material the stretching of $\mathrm{O}-\mathrm{H}$ group was disappeared, but there are intense bands characteristic for carboxylate anion (Figure 3). The IR spectroscopy data confirm the covalent binding of naphthalimide 9 to $\mathrm{SnO}_{2}$ nanoparticles.

\section{Investigation of the complex formation} of 4-methoxynaphthalimide $\mathbf{2} \boldsymbol{b}$ containing dithiacrown ether with heavy metal ions

Complex formation of dithiacrown-containing derivative $\mathbf{2 b}$ with $\mathrm{Cu}\left(\mathrm{ClO}_{4}\right)_{2}, \mathrm{AgClO}, \mathrm{Hg}\left(\mathrm{ClO}_{4}\right)_{2}, \mathrm{Ni}\left(\mathrm{ClO}_{4}\right)_{2}$, $\mathrm{Fe}\left(\mathrm{ClO}_{4}\right)_{2}, \mathrm{Zn}\left(\mathrm{ClO}_{4}\right)_{2}, \mathrm{Cd}\left(\mathrm{ClO}_{4}\right)_{2}, \mathrm{Mg}\left(\mathrm{ClO}_{4}\right)_{2}, \mathrm{Ca}\left(\mathrm{ClO}_{4}\right)_{2}$ was studied in acetonitrile solution and in water. The former observation of naphthalimide containing oxocrown ether in $\mathrm{N}$-aryl moiety ${ }^{[23]}$ by X-ray crystallography method showed

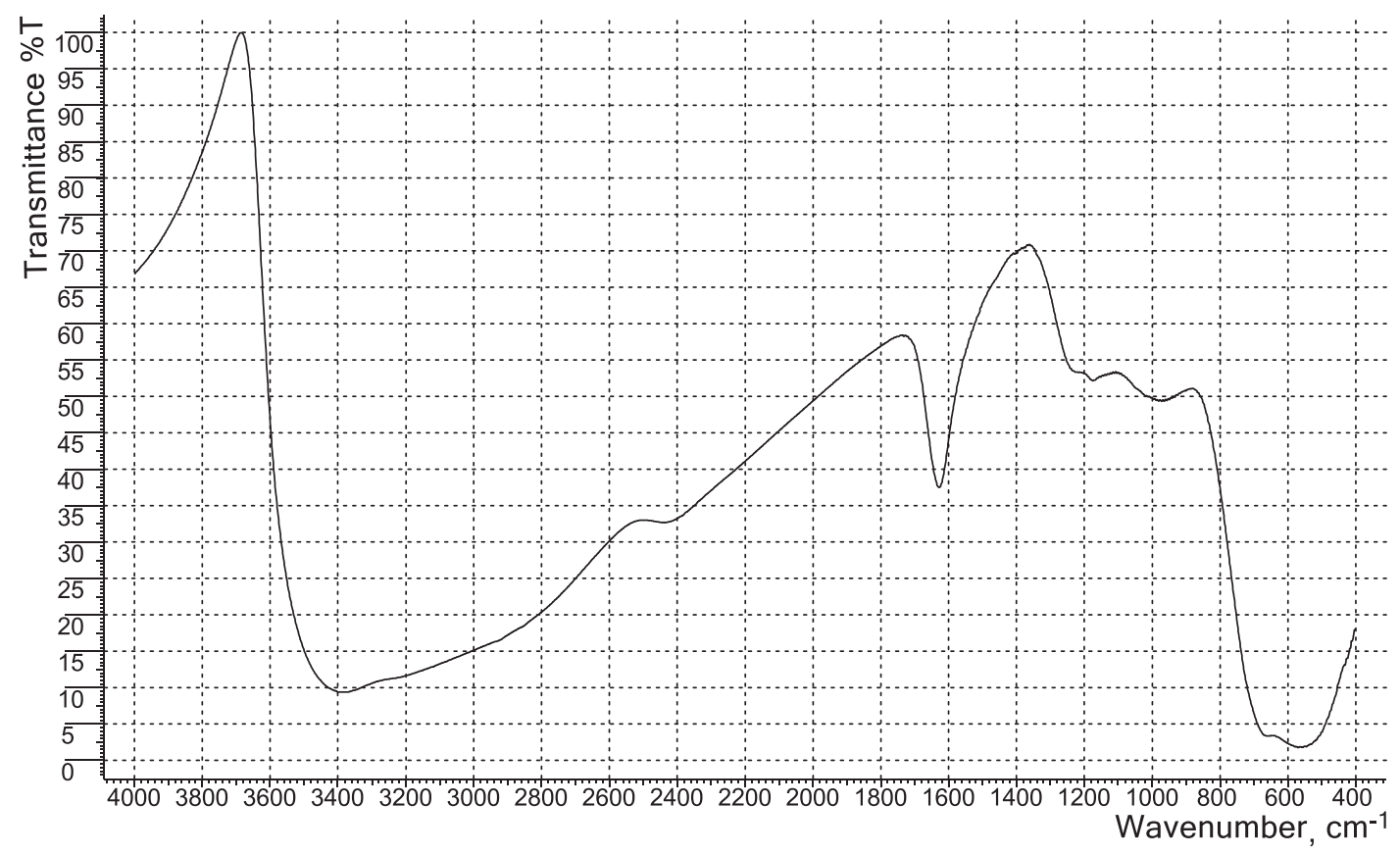

Figure 1. IR spectrum of unmodified $\mathrm{SnO}_{2}$ nanoparticles. 


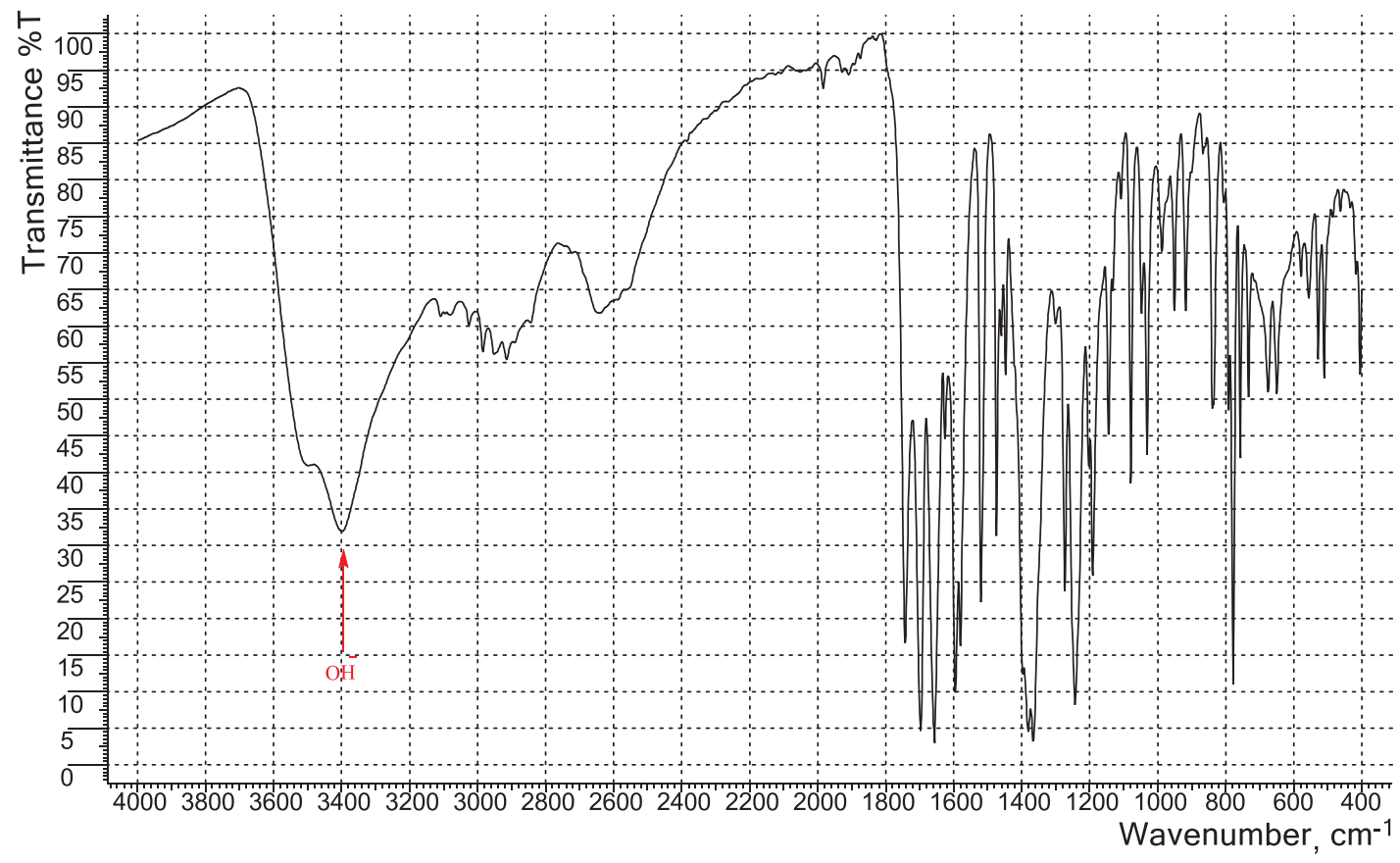

Figure 2. IR spectrum of molecular naphthalimide 9.

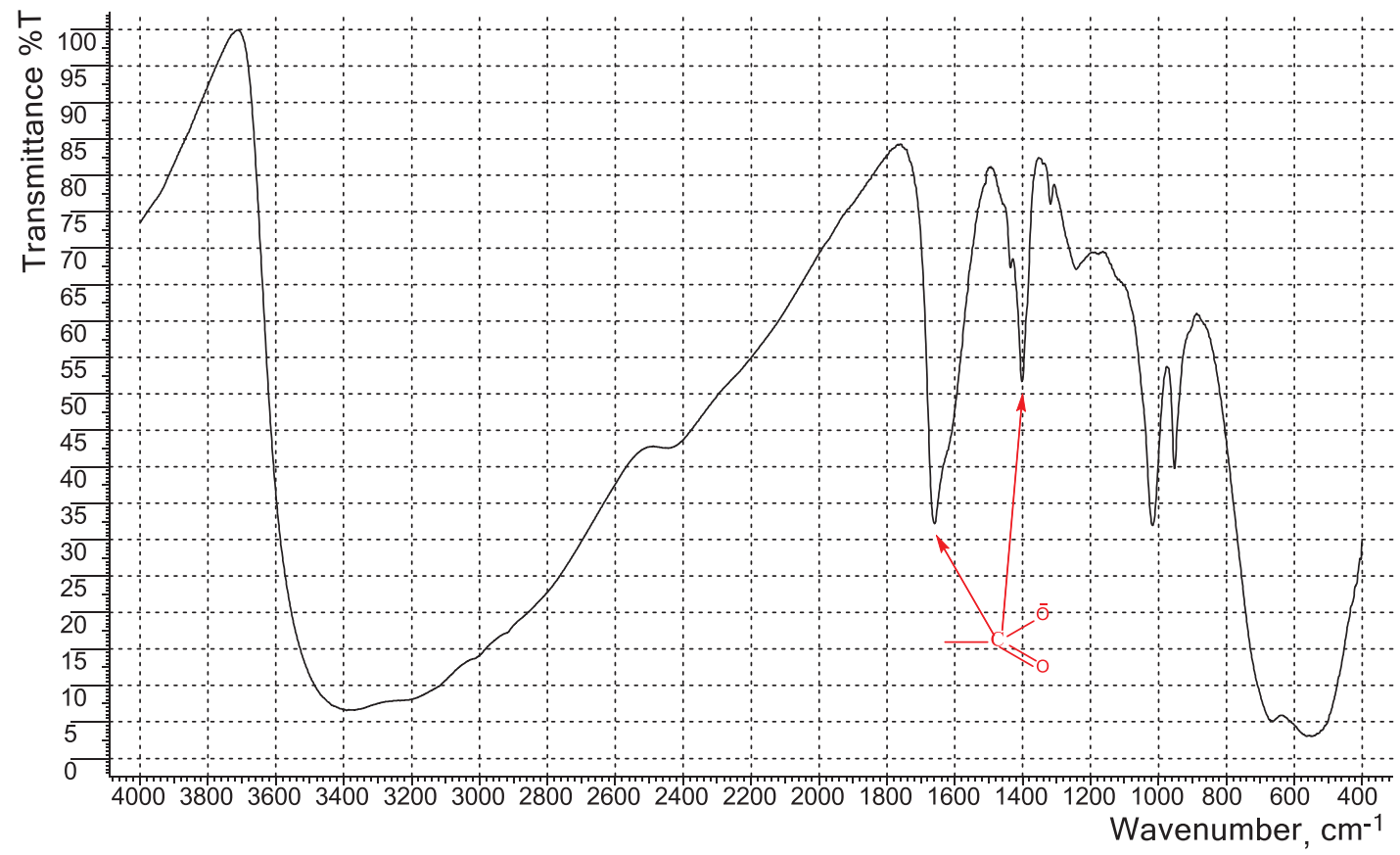

Figure 3. IR spectrum of $\mathrm{SnO}_{2}$ nanoparticles modified by naphthalimide 9.

the dihedral angle between the naphthalimide moiety and $N$-aryl group to be close to $90^{\circ}$. Thus, one can conclude that the $\pi$-electronic interaction between these two fragments is very weak and negligible in the ground state. As a result, the complex formation through interaction of metal ions with crown ether macrocycle does not lead to significant changes in the positions of absorption and fluorescence bands. It was shown also that the presence of crown ether group in the $N$-aryl moiety of naphthalimide leads to dramatic decrease of emission intensity with respect to crown free $N$-phenylnaphthalimide due to efficient electron transfer between naphthalimide chromophore and receptor unit. ${ }^{[23,24]}$ Upon the addition of metal ions the lone pair of electrons of crown ether receptor's heteroatoms are engaged in cation binding, this suppresses PET process and causes fluorescence enhancement (Scheme 5).

As expected the addition of metals salts $\left(\mathrm{Cu}\left(\mathrm{ClO}_{4}\right)_{2}\right.$, $\mathrm{AgClO}_{4}, \mathrm{Hg}\left(\mathrm{ClO}_{4}\right)_{2}, \mathrm{Ni}\left(\mathrm{ClO}_{4}\right)_{2}, \mathrm{Fe}\left(\mathrm{ClO}_{4}\right)_{2}, \mathrm{Zn}\left(\mathrm{ClO}_{4}\right)_{2}, \mathrm{Cd}\left(\mathrm{ClO}_{4}\right)_{2}$, $\left.\mathrm{Mg}\left(\mathrm{ClO}_{4}\right)_{2}, \mathrm{Ca}\left(\mathrm{ClO}_{4}\right)_{2}\right)$ into solution of $\mathbf{2} \mathbf{b}$ does not cause 


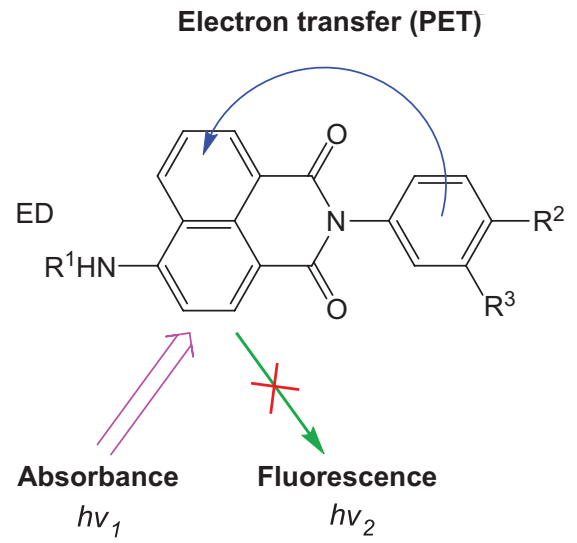

Scheme 5 .

remarkable changes in position of the long wavelength bands in the absorption and fluorescence spectra, but in the case of $\mathrm{Hg}^{2+}, \mathrm{Pb}^{2+}$ and $\mathrm{Cu}^{2+}$ fluorescence enhancement associated with inhibition of photoinduced electron transfer process was observed. Figures $4 \mathrm{a}$, b show the change in absorption and fluorescence spectra of compound $\mathbf{2} \mathbf{b}$ in acetonitrile by adding of 10 equivalents of $\mathrm{Hg}^{2+}, \mathrm{Pb}^{2+}$ and $\mathrm{Cu}^{2+}$ cations. Other studied metal ions did not give rise to remarkable changes in the fluorescence emission even at high concentration. These results are presumably due to well-known high affinity of thiacrown ethers towards the $\mathrm{Hg}^{2+}, \mathrm{Pb}^{2+}$ and $\mathrm{Cu}^{2+}$ cations. ${ }^{[12]}$

The degree of fluorescence enhancement (FE) of $\mathbf{2 b}$ in the presence of tenfold excess of various metal cations is shown in Figure 5. FE value represents the ratio of the integrated fluorescence intensities of the ligand solution in the presence and absence of the cation, respectively. Fluorescent receptor $\mathbf{2 b}$ in acetonitrile solution showed fluorescence enhancement upon addition of $\mathrm{Pb}^{2+}$ cations in 45 times and to a less extent in the presence of $\mathrm{Hg}^{2+}$ (in twenty two times) and $\mathrm{Cu}^{2+}$ (in five times) (Figure 5a). In water compound 2b showed selectivity towards $\mathrm{Hg}^{2+}$ only. The degree of fluorescence enhancement in this case was in eighteen times (Figure 5b).

Studies have shown that the introduction of the two sulfur atoms in the crown ether receptor leads to ligands selective towards the $\mathrm{Hg}^{2+}$ and $\mathrm{Pb}^{2+}$ cations in the presence of alkali, alkaline earth and some heavy metal ions $\left(\mathrm{AgClO}_{4}\right.$, $\mathrm{Ni}\left(\mathrm{ClO}_{4}\right)_{2}, \mathrm{Fe}\left(\mathrm{ClO}_{4}\right)_{2}, \mathrm{Zn}\left(\mathrm{ClO}_{4}\right)_{2}, \mathrm{Cd}\left(\mathrm{ClO}_{4}\right)_{2}, \mathrm{Mg}\left(\mathrm{ClO}_{4}\right)_{2}$, $\left.\mathrm{Ca}\left(\mathrm{ClO}_{4}\right)_{2}\right)$.

\section{Optical properties of $\mathbf{4 a}, \boldsymbol{b}, \mathbf{6}$ and $\boldsymbol{9}$ and nanoparticles of $\mathrm{SnO}_{2}$ modified by $4 \boldsymbol{a}, \boldsymbol{b}, \mathbf{6}$ and $\mathbf{9}$}

Compounds $\mathbf{4 a , b}, \mathbf{6}$ and $\mathbf{9}$ demonstrate the longwavelength absorption band with a maximum at 350 $360 \mathrm{~nm}$ in acetonitrile and $360-375 \mathrm{~nm}$ in water (Figure S7
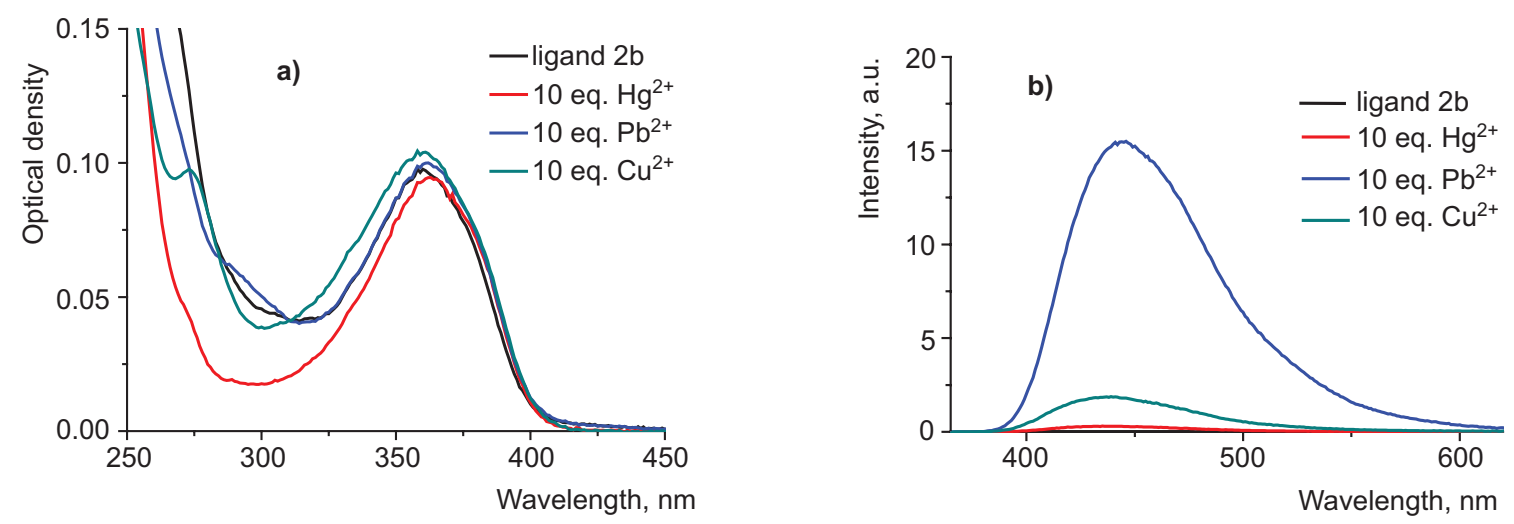

Figure 4. Changes in the absorption spectra (a) and fluorescence (b) of the compound $\mathbf{2} \mathbf{b}$ caused by addition of $\mathrm{Pb}^{2+}, \mathrm{Hg}^{2+}$ and $\mathrm{Cu}^{2+}$ perchlorates in acetonitrile $\left(C_{\mathrm{L}}=6.4 \cdot 10^{-6} \mathrm{~mol} / \mathrm{l}\right)$.
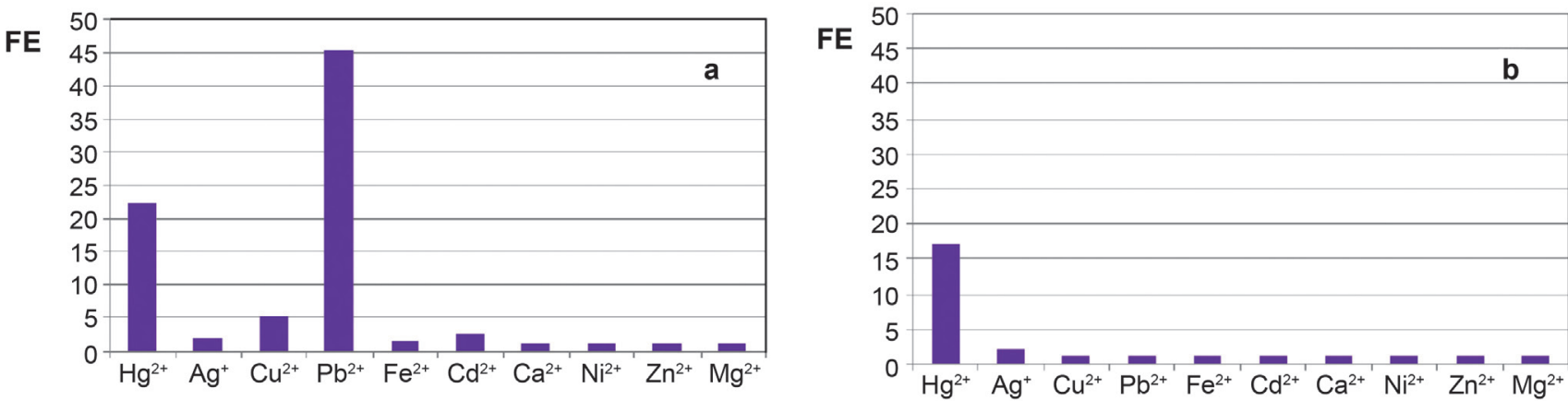

Figure 5. A fluorescence enhancement (FE) of compound $\mathbf{2 b}$ upon addition a tenfold excess of metal perchlorates in acetonitrile (a) and water (b) $\left(C_{\mathrm{L}}=6.4 \cdot 10^{-6} \mathrm{~mol} / \mathrm{l}\right)$. 


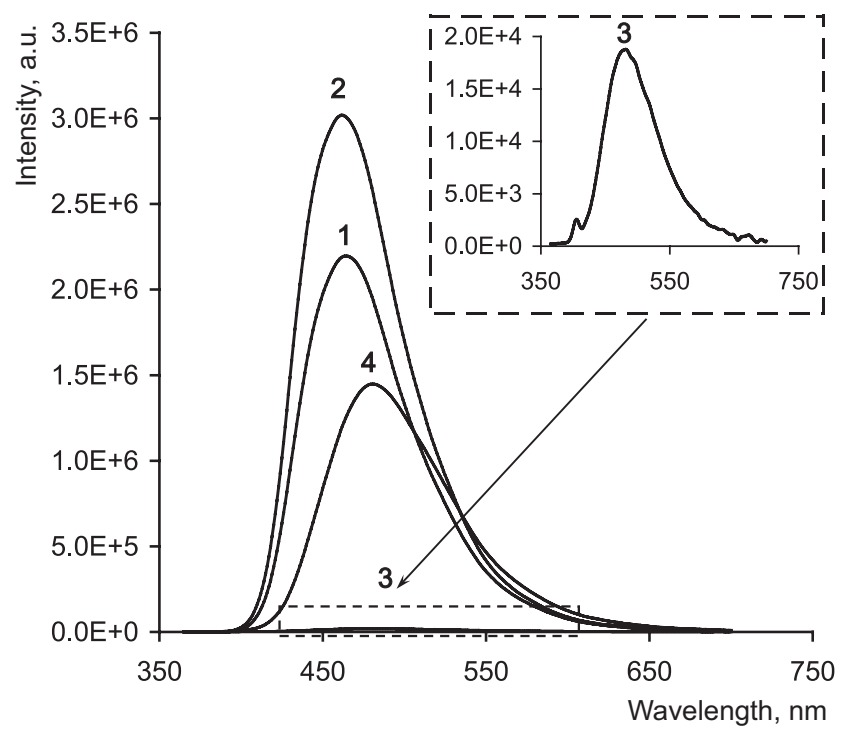

Figure 6. The fluorescence spectra of the compounds 6 (1), 9 (2), 4b (3), 4a (4) in water $\left(C_{\mathrm{L}}=6.4 \cdot 10^{-6} \mathrm{~mol} / \mathrm{l}\right.$, excitation wavelength: $375,375,355,355 \mathrm{~nm}$, respectively).

in SI). Similar to compound $\mathbf{2 b}$ crown containing carboxyl derivative $\mathbf{4 b}$ shows a low level of fluorescence due to the concurrent efficient photoinduced electron transfer process (Figure 6).

It should be noted that the sol of $\mathrm{SnO}_{2}$ nanoparticles does not absorb or emit the light (Figure 7). Analysis of the absorption spectra of $\mathrm{SnO}_{2}$ nanoparticles modified by naphthalimide derivatives shows the absorption in the region 350-360 nm similar to that observed for $\mathbf{4 a , b}, \mathbf{6}$ and $\mathbf{9}$ but of lower intensity (Figures 8 and S8-S10 in SI).

It is important that position and intensity of the fluorescence bands of free $\mathbf{4 a}, \mathbf{b}, \mathbf{6}, \mathbf{9}$ are the same as for those of the compounds in the composition of $\mathrm{SnO}_{2}$ nanoparticles (Figure 9).

The addition of metal salts to the sol $\mathrm{SnO}_{2} \times \mathbf{4 b}$ did not lead to the fluorescence enhancement as observed for molecular ligand $\mathbf{2 b}$. A possible explanation for the observed effect is the assumption that due to considerable length and

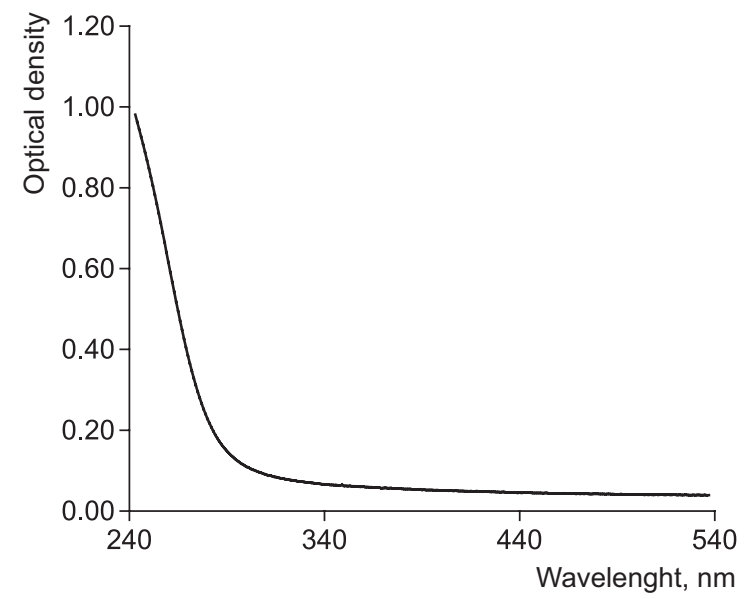

Figure 7. The electronic absorption spectra of unmodified sol of $\mathrm{SnO}_{2}$ nanoparticles in water.

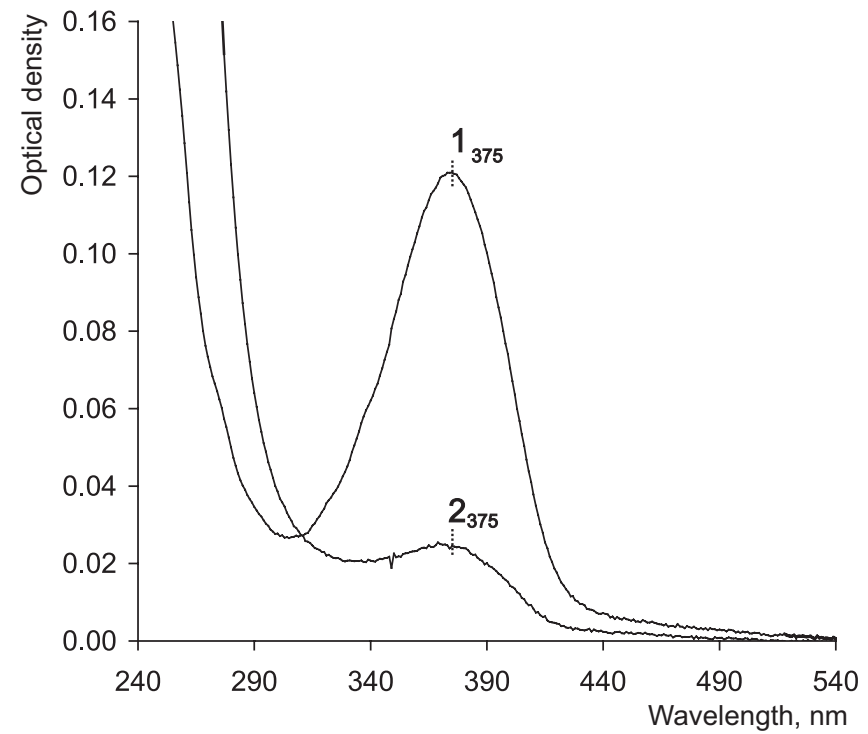

Figure 8. Electronic absorption spectra of compound 6 (1) and modified by it $\mathrm{sol}_{\mathrm{SnO}_{2}} \times \mathbf{6}$ (2) in water.

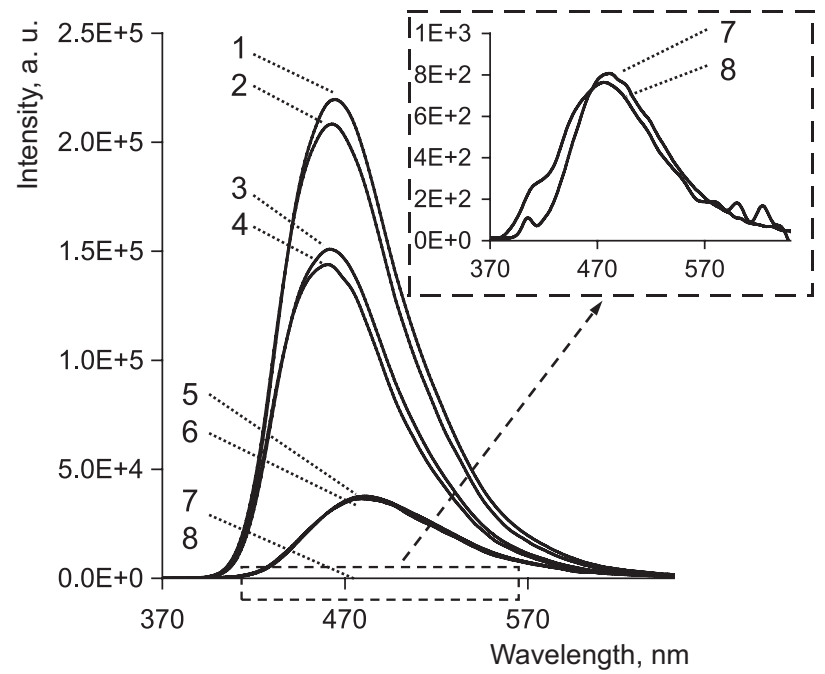

Figure 9. Fluorescence spectra of starting compounds 6 (1), 9 (3), 4a (5), 4b (7) and fluorescence spectra (normalized) of modified sols $\mathrm{SnO}_{2} \times \mathbf{6}(2), \mathrm{SnO}_{2} \times \mathbf{9}(4), \mathrm{SnO}_{2} \times \mathbf{4 a}(6), \mathrm{SnO}_{2} \times \mathbf{4 b}(8)$ in water ( $\mathrm{T}=294{ }^{\circ} \mathrm{K}$, excitation wavelength: $375 \mathrm{~nm}$ for (1)-(4) and $355 \mathrm{~nm}$ for (5)-(8)).

flexibility of the carboxyl-containing spacer the molecule may be placed on the surface of nanoparticles making inaccessible crown ether cavity for the coordination with the metal cations (Scheme 4).

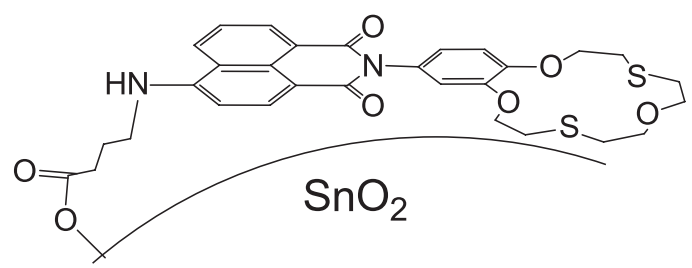

Scheme 4.

Макрогетерочиклы / Macroheterocycles 2017 10(1) 84-93 


\section{Conclusion}

In this paper the synthesis of a number of $N$-alkylor $N$-phenyl-1,8-naphthalimide derivatives having in its structure a carboxylic group and dithiacrown ether as substituents or two substituents of this type simultaneously was represented. Complex formation study of the molecular naphthalimide-containing dithiacrown ether showed selectivity towards $\mathrm{Hg}^{2+}$ and $\mathrm{Pb}^{2+}$ cations in acetonitrile and $\mathrm{Hg}^{2+}$ cations in the water. Influence of the solvent on the ligand selectivity upon complexation is well explained if we take into account the fact that there is a metal cation removing from the solvation shell to the macrocyclic cavity in the process of complex formation. Due to the photoinduced electron transfer process between benzodithiacrown ether receptor and naphthalimide residue in the excited state, free ligands $\mathbf{2 b}, \mathbf{4 b}$ have a low fluorescence intensity and show a significant fluorescence enhancement upon the binding with $\mathrm{Pb}^{2+}$ and $\mathrm{Hg}^{2+}$ cations.

The paper also proposed a method for naphthalimide covalent binding with $\mathrm{SnO}_{2}$ surface. It is shown that carboxyl derivatives as well as acyl chlorides are suitable for this purpose. In the composition of modified $\mathrm{SnO}_{2}$ sols 1,8-naphthalimide derivatives exhibit optical characteristics similar to molecular ones. However, in the case of $\mathrm{SnO}_{2}$ modified by crown-containing naphthalimide complex formation with metal ions has not been observed. It was assumed that due to considerable length and flexibility of the carboxyl-containing spacer the molecule may be placed on the surface of nanoparticles to prevent the coordination of crown ether's cavity with metal cations. Obviously to obtain nanoparticles capable for coordination of cations it is necessary to modify a spacer between the fluorescent receptor and the nanoparticle surface so that the adsorption of the fluorophore on the surface could be impossible.

Acknowledgement. This work was supported by the Agreement №14.616.21.0037 (RFMEFI61615X0037) with Russian Ministry of Education and Sciences.

\section{References}

1. Le T.P., Rogers J.E., Kelly L.A. J. Phys. Chem. A 2000, 104, 6778-6785.

2. Cho D.W., Fujitsuka M., Sugimoto A., Yoon U.C., Mariano P.S., Majima T. J. Phys. Chem. B 2006, 110, 11062-11068.
3. May B., Poteau X., Yuan D., Brown R.G. Dyes Pigm. 1999, 42, 79-84.

4. a) Riu J., Maroto A., Rius F.X. Talanta 2006, 69, 288-301; b) Zhang X. Electrochemical Sensors, Biosensors and their Biomedical Applications Amsterdam, The Netherlands: Elsevier Inc. 2008; c) Holder E., Tessler N., Rogach A.L. J. Mater. Chem. 2008, 18, 1064-1078.

5. Krasovitsky B.M., Zubanova T.P., Vinetskaya Y.M. Chem. Heterocyclic Comp. 1982, 9, 1248-1250.

6. Gruzinskii V.V., Kukhto A.V., Shakkakh G.K. J. Appl. Spectrosc. 1998, 65, 463-465.

7. Siegers C., Olàh B., Würfel U., Hohlebinger J., Hinsch A., Haag R. Solar Energ. Mater. Solar Cells 2009, 93, 552-563.

8. Lin H.-H., Chan Y.-C., Chen J.-W., Chang C.-C. J. Mater. Chem. 2011, 21, 3170-3177.

9. Magalhães J.L., Pereira R.V., Triboni E.R., Filho P.B., Gehlen M.H., Nart F.C. Photochem. Photobiol. A 2006, 183, 165-170.

10. Li Z., Yang Q., Qian X. Bioorg. Med. Chem. 2005, 13, 4864 4870.

11. Batzill M., Diebold U. Prog. Surf. Sci. 2005, 79, 47-154.

12. Alfimov M.V., Gromov S.P., Fedorov Yu.V., Fedorova O.A., Vedernikov A.I., Churakov A.V., Kuzmina L.G., Howard J.A.K., Bossmann S., Braun A., Woerner M., Sears D.F., Saltiel J. J. Am. Chem. Soc. 1999, 121, 4992-5000.

13. Panchenko P.A., Fedorov Y.V., Fedorova O.A., Izmailov B.A., Vasnev V.A., Istratov V.V., Makeeva E.A., Rumyantseva M.N., Gaskov A.M. Mendeleev Commun. 2011, 21, 12-14.

14. Nardis S., Monti D., Di Natale C., D'Amico A., Siciliano P., Forleo A., Epifani M., Taurino A., Rella R., Paolesse R. Sens. Actuators B 2004, 103, 339-343.

15. Callone E., Carturan G., Ischia M., Epifani M., Forleo A., Siciliano P., Paolesse R. Inorg. Chim. Acta 2008, 361, 79-85.

16. Descalzo A.B., Martinez-Manez R., Radeglia R., Rurack K., Soto J. J. Am. Chem. Soc. 2003, 125, 3418-3419.

17. Okazaki M., Suhara Y., Uemura S., Fujiyama M., Oda K., Tanaka T., Taniguchi S., Watanabe Y. J. Soc. Organ. Synth. Chem., Jpn. 1956, 14, 455-459.

18. Okazaki M., Suhara Y., Oda K. J. Soc. Organ. Synth. Chem., Jpn. 1956, 14, 504-508.

19. Reynolds G.A., Drexhage K.H. Optics Commun. 1975, 13, 222-225.

20. Lisitsyn V.N. Chemistry and Technology of Intermediates. Moscow: Khimiya, 1987. 368 p. (in Russ.) [Лисицын B.H. Химия и технология промежуточных продуктов. М. Химия, 1987. 368 с.].

21. Cao H., Chang V., Hernandez R., Heagy M.D. J. Org. Chem. 2005, 70, 4929-4934.

22. Silverstein P., Bassler G., Morril T. Spectrometric Identification of Organic Compounds. New York: Wiley, 1977. 183 p.

23. Panchenko P.A., Fedorov Yu.V., Perevalov V.P., Jonusauskas G., Fedorova O.A. J. Phys. Chem. A 2010, 114, 4118-4122.

24. Panchenko P.A., Fedorov Y.V., Fedorova O.A., Perevalov V.P., Jonuskauskas G. Russ. Chem. Bull. 2009, 6, 1199-1206. 